Conflict of interest: S.J. Draper is a named inventor on patent applications relating to $\mathrm{RH} 5$ and/or other malaria vaccines and immunization regimens is a cofounder of, shareholder in, and consultant for SpyBiotech; and declares research funding support from Pfizer and GSK BioPharm. A.D. Douglas, G.J. Wright, and A.V.S. Hill are named inventors on patent applications relating to RH5 and/or other malaria vaccines and immunization regimens. L. Siani and S. Di Marco are employees of ReiThera (formerly Okairos), which is currently developing vectored vaccines for a number of diseases. J. Vekemans was an employee of CSK, which has acquired the ChAd63 vector from Okairos. R. Ashfield is a director of Ducentis and holds shares in the company, which is developing a therapy for autoimmune disease. A.M. Minassian has an immediate family member who is an inventor on patents relating to $\mathrm{RH} 5$ and/or other malaria vaccines and immunization regimens and who is a cofounder of, shareholder in, and consultant for SpyBiotech. S. Biswas is a cofounder and CEO of, and shareholder in, SpyBiotech and is a contributor in a patent application relating to multimerisation technology. J. Jin is a cofounder of and shareholder in SpyBiotech.

License: This work is licensed under the Creative Commons Attribution 4.0 International License. To view a copy of this license, visit http:// creativecommons.org/licenses/by/4.0/.

Submitted: July 31, 2017

Accepted: October 5, 2017

Published: $x x x x$

Reference information:

JCI Insight. 2017;2(21):e96381.

https://doi.org/10.1172/jici.

insight.96381.

\section{Human vaccination against RH5 induces neutralizing antimalarial antibodies that inhibit RH5 invasion complex interactions}

Ruth O. Payne, ${ }^{1}$ Sarah E. Silk, ${ }^{1}$ Sean C. Elias, ${ }^{1}$ Kazutoyo Miura, ${ }^{2}$ Ababacar Diouf, ${ }^{2}$ Francis Galaway, ${ }^{3}$ Hans de Graaf, ${ }^{4}$ Nathan J. Brendish, ${ }^{4}$ Ian D. Poulton, ${ }^{1}$ Oliver J. Griffiths, ${ }^{1}$ Nick J. Edwards, ${ }^{1}$ Jing Jin, ${ }^{1}$ Geneviève M. Labbé, ${ }^{1}$ Daniel G.W. Alanine, ${ }^{1}$ Loredana Siani, ${ }^{5}$ Stefania Di Marco, ${ }^{5}$ Rachel Roberts, ${ }^{1}$ Nicky Green, ${ }^{6}$ Eleanor Berrie, ${ }^{6}$ Andrew S. Ishizuka, ${ }^{1}$ Carolyn M. Nielsen, ${ }^{1}$ Martino Bardelli, ${ }^{1}$ Frederica D. Partey, ${ }^{1,7,8}$ Michael F. Ofori, ${ }^{8}$ Lea Barfod, ${ }^{1}$ Juliana Wambua, ${ }^{9}$ Linda M. Murungi, ${ }^{1,9}$ Faith H. Osier, ${ }^{9}$ Sumi Biswas, ${ }^{1}$ James S. McCarthy, ${ }^{10}$ Angela M. Minassian, ${ }^{1}$ Rebecca Ashfield, ${ }^{1}$ Nicola K. Viebig, ${ }^{11}$ Fay L. Nugent, ${ }^{1}$ Alexander D. Douglas, ${ }^{1}$ Johan Vekemans, ${ }^{12}$ Gavin J. Wright, ${ }^{3}$ Saul N. Faust, ${ }^{4}$ Adrian V.S. Hill, ${ }^{1}$ Carole A. Long, ${ }^{2}$ Alison M. Lawrie, ${ }^{1}$ and Simon J. Draper ${ }^{1}$

'The Jenner Institute, University of Oxford, Oxford, United Kingdom. 'Laboratory of Malaria and Vector Research, NIAID/NIH, Rockville, Maryland, USA. ${ }^{3}$ Cell Surface Signalling Laboratory, Wellcome Trust Sanger Institute, Cambridge, United Kingdom. ${ }^{4} \mathrm{NIHR}$ Wellcome Trust Clinical Research Facility, University Hospital Southampton NHS Foundation Trust and Faculty of Medicine, University of Southampton, Southampton, United Kingdom. ${ }^{5}$ ReiThera SRL (formerly Okairos SRL), Viale Città d'Europa, Rome, Italy. ${ }^{6}$ Clinical Biomanufacturing Facility, University of Oxford, Oxford, United Kingdom. ${ }^{7}$ Centre for Medical Parasitology, Department of Immunology and Microbiology (ISIM), Faculty of Health and Medical Sciences, University of Copenhagen, Copenhagen, Denmark. ${ }^{8}$ Department of Immunology, Noguchi Memorial Institute for Medical Research, University of Chana, Legon, Chana. ${ }^{9}$ KEMRI Centre for Geographic Medicine Research, Kilifi, Kenya. ${ }^{10}$ QIMR Berghofer Medical Research Institute, Herston, Queensland, Australia. "'European Vaccine Initiative, UniversitätsKlinikum Heidelberg, Heidelberg, Germany. ${ }^{12}$ GSK Vaccines, Wavre, Belgium.

The development of a highly effective vaccine remains a key strategic goal to aid the control and eventual eradication of Plasmodium falciparum malaria. In recent years, the reticulocyte-binding protein homolog 5 (RH5) has emerged as the most promising blood-stage $P$. falciparum candidate antigen to date, capable of conferring protection against stringent challenge in Aotus monkeys. We report on the first clinical trial to our knowledge to assess the RH5 antigen - a dose-escalation phase la study in $\mathbf{2 4}$ healthy, malaria-naive adult volunteers. We utilized established viral vectors, the replication-deficient chimpanzee adenovirus serotype 63 (ChAd63), and the attenuated orthopoxvirus modified vaccinia virus Ankara (MVA), encoding RH5 from the 3D7 clone of $P$. falciparum. Vaccines were administered i.m. in a heterologous prime-boost regimen using an 8-week interval and were well tolerated. Vaccine-induced anti-RH5 serum antibodies exhibited cross-strain functional growth inhibition activity (GIA) in vitro, targeted linear and conformational epitopes within RH5, and inhibited key interactions within the RH5 invasion complex. This is the first time to our knowledge that substantial RH5-specific responses have been induced by immunization in humans, with levels greatly exceeding the serum antibody responses observed in African adults following years of natural malaria exposure. These data support the progression of RH5-based vaccines to human efficacy testing.

\section{Introduction}

The most severe form of human malaria is caused by the Plasmodium falciparum parasite. Despite recent and encouraging advances in malaria control measures, current estimates suggest that, in 2015, there were still over 200 million clinical cases leading to 438,000 deaths (1). Consequently, the development of an effective and durable vaccine remains a key strategic goal to aid the control, local elimination, and eventual eradication of this disease (2). The mainstay approach to vaccination against the blood-stage of malaria infection is to induce antibodies against the merozoite form of the parasite that invades erythrocytes (3). Such a vac- 
cine would protect against disease severity and could reduce transmission by accelerating the control and clearance of blood-stage parasitemia, either in a standalone manner or by complementing preerythrocytic immunity induced by partially effective subunit (4) or whole sporozoite vaccine strategies (5).

However, despite the fact that naturally acquired immunity to malaria is largely directed against the blood-stage parasite (6), the development of an effective vaccine has proved exceptionally challenging. The most widely studied merozoite candidate vaccine antigens have been hindered by substantial levels of polymorphism (7) alongside redundant erythrocyte invasion pathways (8). Extremely high concentrations of functional antibody against these antigens have also been required to neutralize the parasite (9), likely linked to the kinetic constraints imposed by very rapid erythrocyte invasion (10). Nevertheless, substantial progress has been made in recent years, with the identification of a new generation of merozoite antigen targets that are essential and that exhibit relatively low levels of polymorphism, leading to induction of strain-transcending antibodies by vaccination in preclinical models (11). Three of these targets form a complex, including the $P$. falciparum reticulocyte-binding protein homolog 5 (RH5) (12), the cysteine-rich protective antigen (CyRPA) (13), and the RH5-interacting protein (Ripr) (14). Of these, vaccine development efforts are currently most advanced for RH5 (15).

The first vaccination studies with RH5 used regions of the antigen made in $E$. coli that failed to induce functional antibodies $(16,17)$. However, functional neutralizing antibodies were subsequently generated by using immunogens based on the full-length RH5 (RH5_FL) sequence. These antibodies raised by vaccination of animals can cross-inhibit all $P$. falciparum lines and field isolates tested to date $(12,18-20)$, notably with higher efficiency than other historical target antigens (18). RH5 is also essential $(16,21)$ and forms a critical nonredundant interaction with its receptor basigin (CD147) on the RBC surface (22). Somewhat surprisingly, RH5 appears to be under relatively low-level immune pressure following natural infection $(12,23-26)$, with functional constraints also linked to basigin binding and host RBC tropism $(21,27,28)$ - both of these factors potentially explain its relatively high degree of sequence conservation. Recently, the N-terminal region of RH5 (RH5Nt) has been shown to bind the essential glycosylphosphatidylinositol-anchored (GPI-anchored) merozoite protein P113, providing a mechanism for anchoring RH5 to the merozoite surface (29). When bound by P113, RH5_FL is able to bind basigin and CyRPA, but not Ripr. CyRPA, however, can bind Ripr and likely recruits this molecule to the complex leading to its one-way release from P113, either by proteolytic cleavage of RH5Nt or mutually exclusive binding of P113 and Ripr. The function of the RH5-CyRPA-Ripr complex has been linked to pore formation between the merozoite and the RBC, allowing for the movement of $\mathrm{Ca}^{2+}$ into the host cell and alterations in $\mathrm{RBC}$ cytoskeleton architecture, prior to establishment of the tight junction $(30,31)$.

The traditional approach to antibody induction by vaccination has been the delivery of recombinant protein, or particle-in-adjuvant formulations. An alternative strategy, developed over the last decade, has seen the use of recombinant viral vectored vaccines to deliver protein antigens of interest, whereby the design and administration of these vectors has been optimized to induce antibodies in conjunction with the $\mathrm{T}$ cell responses for which they were historically favored $(32,33)$. Such a strategy is particularly attractive when the antigen of interest is refractory to production in recombinant form using heterologous expression platforms. Indeed, the RH5_FL protein proved particularly difficult to express for many years, with the first highly promising vaccine data generated by use of the viral vector platform (12). In the case of this approach, a recombinant replication-deficient adenovirus (of human or simian serotype) is used to prime the immune response, followed by a booster vaccination (typically 8 weeks later) with an attenuated poxvirus recombinant for the same antigen (33). This heterologous prime-boost approach has shown antibody induction against difficult-to-express malaria antigens in numerous animal models, including nonhuman primates $(32,34,35)$. These vectors, delivering antigens from $P$. falciparum, have now been shown to be safe and immunogenic for $\mathrm{T}$ cell and antibodies in healthy European and American adult volunteers (36-40), as well as African adults, children, and infants $(41,42)$. More recently, similar adenovirus-poxvirus vectored vaccine technologies have been used to immunize humans against numerous other pathogens including $P$. vivax malaria (43), Ebola virus (44), hepatitis C virus (HCV) (45), respiratory syncytial virus (RSV) (46) and HIV-1 (47).

Here, we report the safety and immunogenicity of a replication-deficient chimpanzee adenovirus serotype 63 (ChAd63) and an attenuated orthopoxvirus modified vaccinia virus Ankara (MVA) encoding monovalent RH5_FL based on the sequence from the 3D7 clone of $P$. falciparum. These vaccines were tested in an open-label dose-escalation phase Ia study in healthy United Kingdom (UK) adults. These vaccines have been previously shown to be immunogenic in mice and rabbits (12) and exhibited significant in vivo protec- 


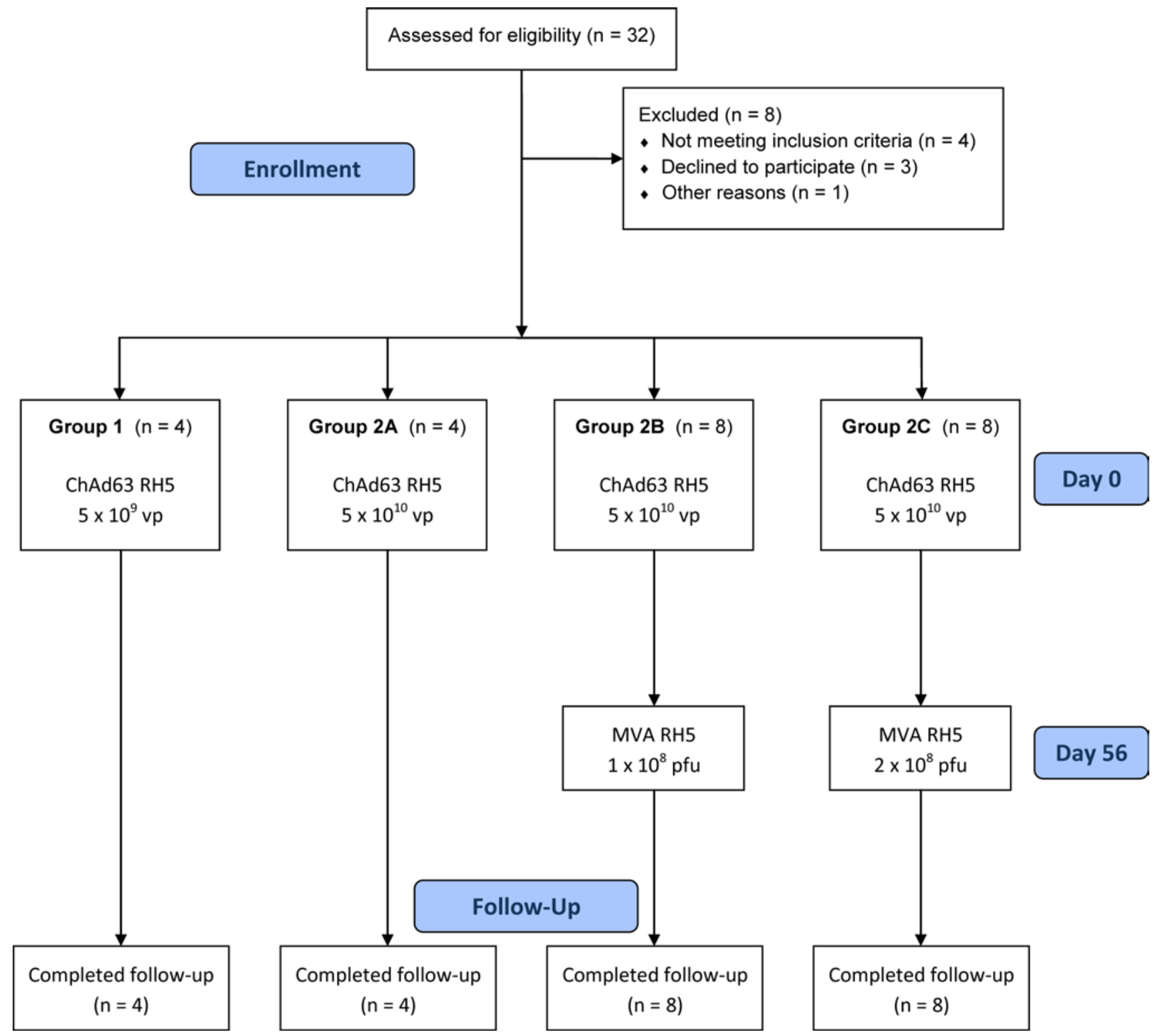

Figure 1. VAC057 flow chart of study design and volunteer recruitment. Enrolment into the VAC057 study began in August 2014, and all follow-up visits were completed by October 28, 2015. All immunizations were administered i.m. into the deltoid of the nondominant arm preferentially.

tion against a stringent heterologous strain blood-stage P. falciparum challenge in Aotus monkeys (34). Protection was strongly associated with anti-RH5_FL serum IgG antibody concentration and in vitro growth inhibition activity (GIA) measured using purified IgG (34). Now, we report that these vaccines demonstrate a favorable safety profile in malaria-naive adults and confirm for the first time to our knowledge that substantial RH5-specific antibodies, B cell, and T cell responses can be induced by immunization in humans. Vaccine-induced serum antibodies exhibited cross-strain in vitro GIA, targeted linear and conformational epitopes within RH5, and inhibited key interactions within the RH5 invasion complex, including those between RH5 and P113, CyRPA and basigin.

\section{Results}

Twenty-four healthy adult volunteers were enrolled into the VAC057 trial to assess the ChAd63-MVA RH5 vaccine in an open-label, dose-escalation study design. Thirty-two volunteers were screened in total, across 2 sites, and 24 of these were enrolled (Figure 1). Vaccinations began on August 18, 2014, and all follow-up visits were completed by October 28, 2015. All vaccinees received their immunizations as scheduled, and there were no withdrawals from the study. Similar numbers of males and females were enrolled (13 females, 11 males). 
A

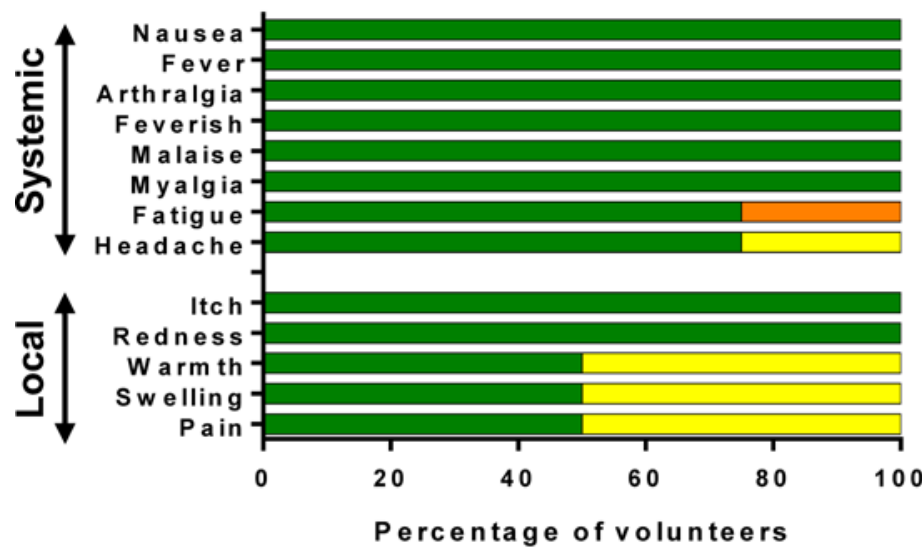

C

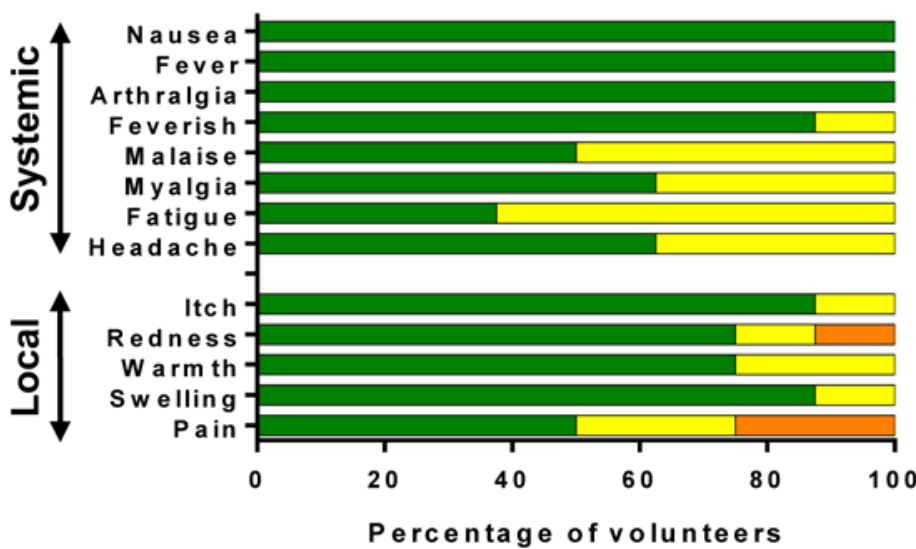

None $\square$ Mild
B

Group 2 post-ChAd 63 RH 5

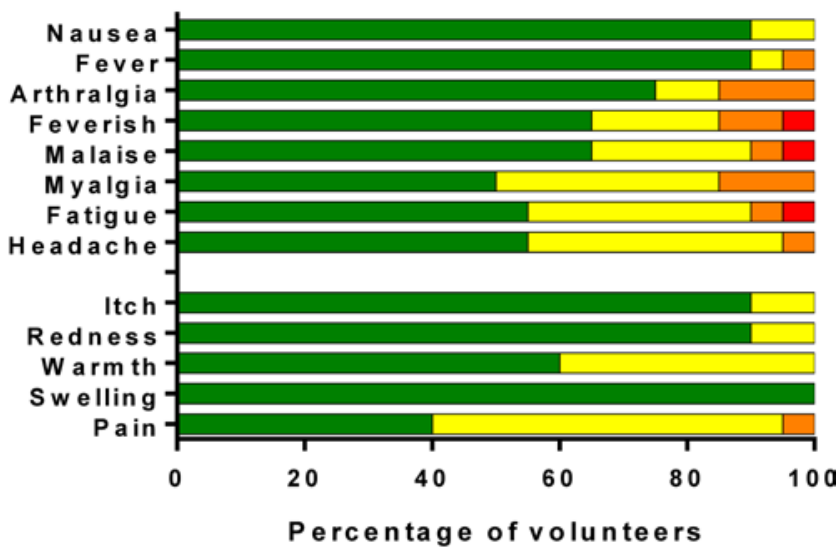

D

Group 2 C post-MVA RH 5

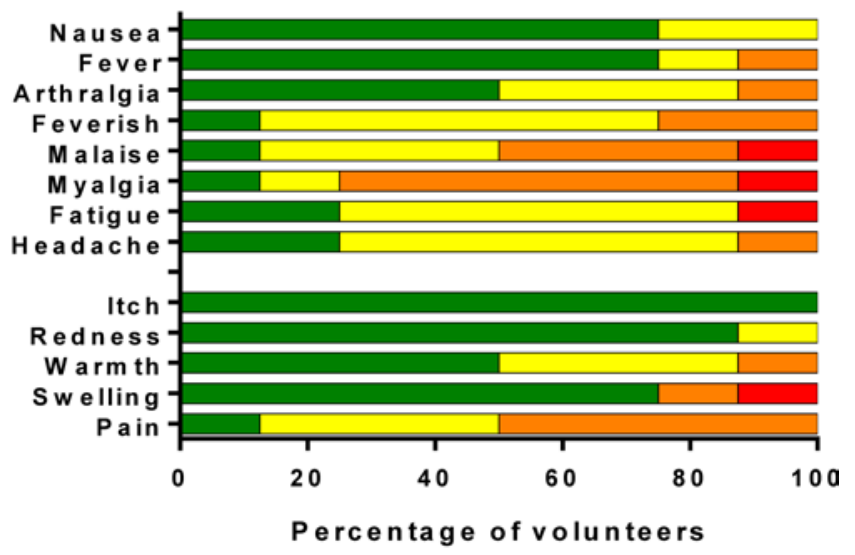

Moderate

\section{Severe}

Figure 2. Solicited AEs following vaccination with ChAd63 and MVA RH5. The solicited local and systemic adverse events (AEs) recorded for 7 days following ChAd63 RH5 and MVA RH5 are shown at the maximum severity reported by all volunteers. (A) Four volunteers received $5 \times 10^{9}$ viral particles (vp) ChAd63 RH5 (Group 1), and (B) 20 received $5 \times 10^{10} \mathrm{vp}$ (Group 2). (C) Eight of the Group 2 volunteers went on to receive MVA RH5 $1 \times 10^{8}$ plaque-forming units (pfu) (Group 2B), and (D) 8 received $2 \times 10^{8}$ pfu (Group 2C).

The mean age of volunteers was 28 years (range 19-48 years). The 4 Group 1 volunteers received $5 \times 10^{\circ}$ viral particles (vp) of the ChAd63 RH5 vaccine. Following a safety review, the dose of ChAd63 RH5 was increased for Group 2 and volunteers received $4.26 \times 10^{10}$ to $4.77 \times 10^{10} \mathrm{vp}$ (a nominal figure of $5 \times 10^{10} \mathrm{vp}$ is otherwise used throughout this report). Four volunteers in Group 2A received ChAd63 RH5 alone. Eight volunteers were enrolled into Group 2B, and a further 8 were enrolled into Group 2C. These volunteers received ChAd63 RH5, followed 8 weeks later with a boost vaccination of MVA RH5 at a dose of $1 \times 10^{8}$ plaque-forming units (pfu) (Group 2B) or $2 \times 10^{8}$ pfu (Group 2C). There was a 2-week interval between the final vaccination in Group 2B with MVA RH5 at the lower dose of $1 \times 10^{8}$ pfu and the first vaccination with MVA RH5 at the full dose of $2 \times 10^{8}$ pfu in Group 2C, with a safety review prior to dose escalation.

ChAd63 and MVA RH5 show a favorable safety profile in healthy UK adult volunteers. There were no serious adverse events (SAEs) or unexpected reactions and no safety concerns during the course of the trial. The reactogenicity of the vaccines was similar to that seen in previous malaria vaccine trials using the same viral vectors at similar doses in healthy adults $(37,43,48)$, with the higher doses of both vaccines associated with an increased number and higher severity of reported AEs (Figure 2). The majority of AEs following ChAd63 RH5 were mild, but moderate AEs were reported by some volunteers in both groups, and 2 volunteers who received the full dose reported severe AEs on the day of vaccination that resolved within 24 hours. All moderate or severe solicited systemic AEs following MVA RH5 occurred in volunteers who had received the higher dose of vaccine. The 
majority of solicited AEs occurred within the first 2 days after vaccination, and the median duration of each systemic $\mathrm{AE}$ was between 1 and 2 days following either vaccine. The unsolicited AEs considered possibly, probably, or definitely related to either vaccine are shown in Supplemental Table 1 (supplemental material available online with this article; https://doi.org/10.1172/jci.insight.96381DS1). The majority were mild in nature, and all resolved spontaneously. There were no severe laboratory AEs following ChAd63/MVA RH5 vaccination. One volunteer had a moderately raised ALT (123 IU/1) at day 7 (d7) following ChAd63 RH5 that had resolved fully by d28. One volunteer had moderate thrombocytopenia $\left(123 \times 10^{9} / 1\right)$ and mild leukopenia $\left(3.3 \times 10^{9} / 1\right)$ at $\mathrm{d} 28$ following ChAd63 RH5 but had commenced postexposure prophylaxis for a possible HIV exposure the day before these bloods were taken; therefore, causality is unclear. All other laboratory AEs were mild and had resolved fully by $\mathrm{d} 84$, except for 1 volunteer who had a persistent mild anemia. This had been present at screening and had not worsened over the course of the study, so it was not considered significant.

ChAd63 and MVA RH5 expand IFN- $\gamma$ T cell responses in healthy UK adult volunteers. The kinetics and magnitude of the RH5-specific T cell response were assessed over time by ex vivo IFN- $\gamma$ ELISPOT following restimulation of PBMC with 20-mer peptides overlapping by 10 amino acids (aa) spanning the entire RH5 insert present in the vaccines (Figure 3 and Supplemental Table 2). Vaccination with ChAd63MVA RH5 induced antigen-specific T cell responses in all volunteers, with individual responses shown in Supplemental Figure 1 and median responses to the total vaccine insert shown for each group in Figure 3A. Following ChAd63 RH5 prime, there was no significant difference between median responses in the lower-dose Group 1 in comparison with Group 2 at the peak of the response on d14 (median 499 [range 96-993] vs. 797 [range 3-3,269] spot forming units [SFU]/million PBMC in Groups 1 vs. 2 respectively [ $n=4$ vs. $20, P=0.27$ by Mann-Whitney test]) (Figure 3B). Responses subsequently followed classical $\mathrm{T}$ cell kinetics and contracted by d56 (Figure 3A). Administration of MVA RH5 significantly boosted these responses in all volunteers as measured 1 week later on d63 (Groups 2B and 2C vs. 2A, Kruskal-Wallis test with Dunn's multiple comparison test) (Figure 3C), reaching medians of 2092 [range 628-4,102] and 2,281 [range 935-4,257] SFU/million PBMC in Groups 2B and 2C, respectively, vs. 213 [range 15-363] $\mathrm{SFU} /$ million PBMC in Group 2A. However, there was no significant difference between the 2 groups who received the different doses of MVA RH5 $(P=0.33$, Mann-Whitney test). T cell responses were detected in all 6 of the peptide pools used in the ELISPOT assay (Supplemental Figure 2), confirming these were spread across the whole RH5_FL antigen, and thus including both structured and disordered regions of the molecule $(49,50)$. Following the peak at $d 63$, responses contracted and were maintained above baseline at the end of the study period, again with no significant difference between Groups $2 \mathrm{~B}$ and 2C ( $P=0.27$, Mann-Whitney test) (Figure 3D).

ChAd63 and MVA RH5 induce serum antibody responses and memory $B$ cells ( $m B C s$ ) in healthy UK adult volunteers. The kinetics and magnitude of the anti-RH5 serum IgG antibody response were assessed over time by ELISA against RH5_FL recombinant protein (Figure 4). Priming vaccination with $5 \times 10^{10} \mathrm{vp}$ ChAd63 RH5 followed by MVA RH5 boost induced antigen-specific IgG responses in all volunteers (Groups 2B and 2C), with individual responses shown in Supplemental Figure 3 and median responses shown for each group in Figure 4A. Responses are reported in $\mu \mathrm{g} / \mathrm{ml}$ following conversion of ELISA arbitrary units (AU) by calibration-free concentration analysis (CFCA) (Supplemental Figure 4). Following ChAd63 RH5 prime with $5 \times 10^{9} \mathrm{vp}, 2$ of 4 volunteers showed a detectable response on $\mathrm{d} 28$, in contrast to 16 of 20 volunteers (median: 0.2 , range: $0-2.3 \mu \mathrm{g} / \mathrm{ml}, n=20$ ) following priming with $5 \times$ $10^{10} \mathrm{vp}(P=0.13$, Mann-Whitney test) (Figure 4B). Responses were subsequently maintained in Group 2 volunteers prior to administration of MVA RH5, which led to a boost as measured 4 weeks later on d84 (Figure 4A); this reached significance for Group 2C vs. 2A ( $P=0.008$, Kruskal-Wallis test with Dunn's multiple comparison test) (Figure 4B). Responses in Group 2C (median: 9.3, range: 0.5-14.5 $\mu \mathrm{g}$ / $\mathrm{ml}, n=8$ ) tended to be higher than in Group $2 \mathrm{~B}$, but this did not reach significance (median: 4.0, range: 2.1-17.5 $\mu \mathrm{g} / \mathrm{ml}, n=8)$ at this peak time point ( $P=0.33$, Mann-Whitney test). Serum antibody responses decreased by $\mathrm{d} 140$ but were well maintained above preboost levels, with significance of Group 2C over 2A ( $P=0.005$, Kruskal-Wallis test with Dunn's multiple comparison test) (Figure 4B). We also compared postboost responses induced by the vaccine (Groups $2 \mathrm{~B}$ and $2 \mathrm{C}$ combined) with those induced by natural malaria exposure in 79 Ghanaian adults and 96 Kenyan adults (Figure 4C). Anti-RH5_FL IgG responses were weak in the malaria-endemic populations, with median responses less than $0.1 \mu \mathrm{g} / \mathrm{ml}$ in both and the highest $4 \mu \mathrm{g} / \mathrm{ml}$ in a single Kenyan adult. The vaccine-induced responses were significantly higher $(P$ $<0.0001$, Kruskal-Wallis test with Dunn's multiple comparison test). 
A

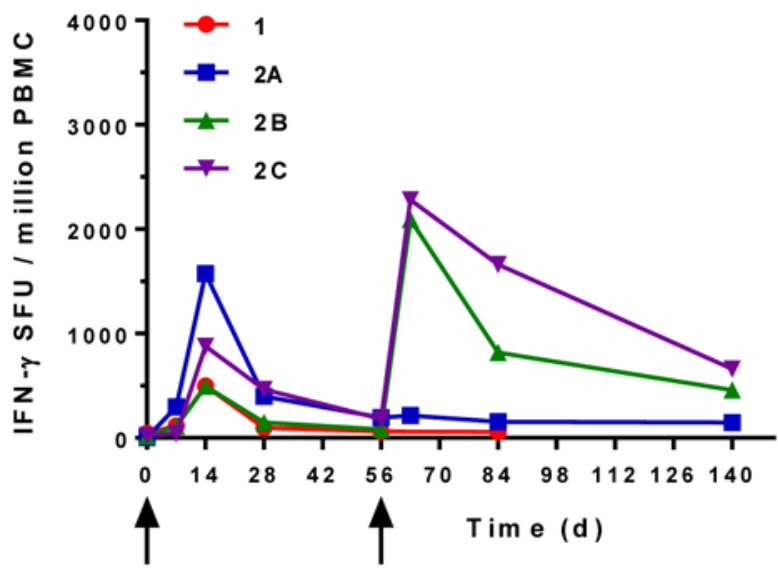

C

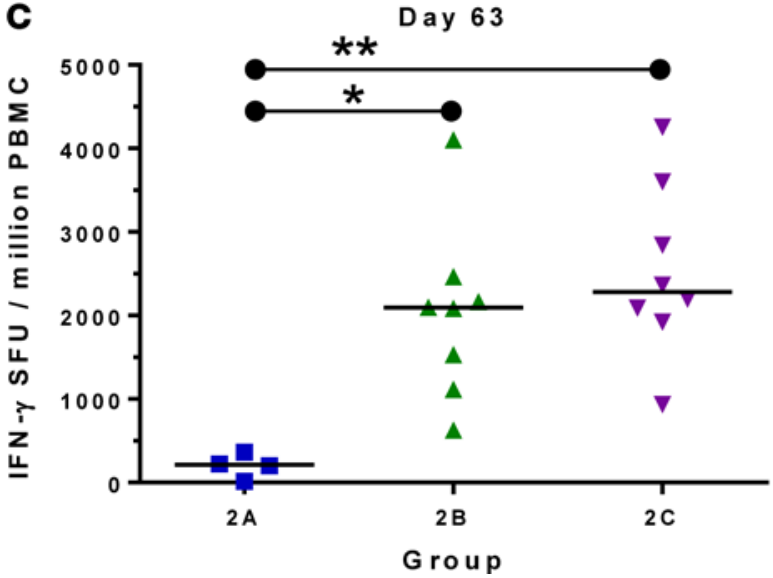

B

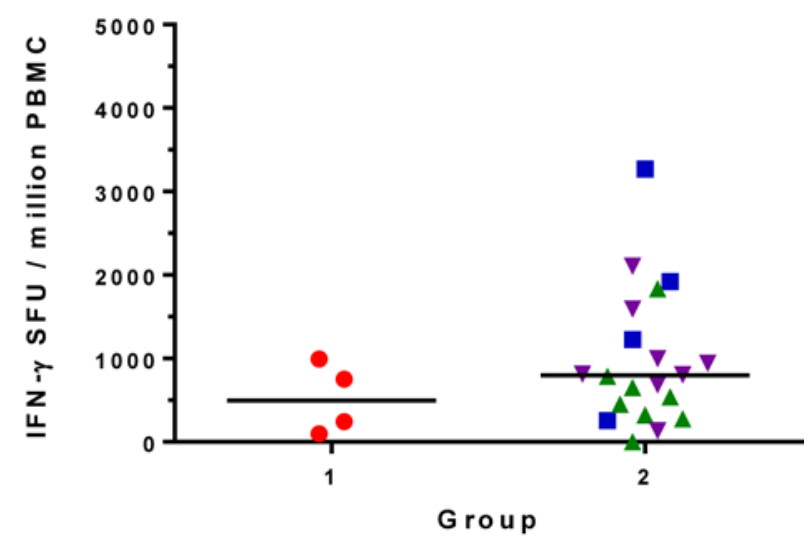

D

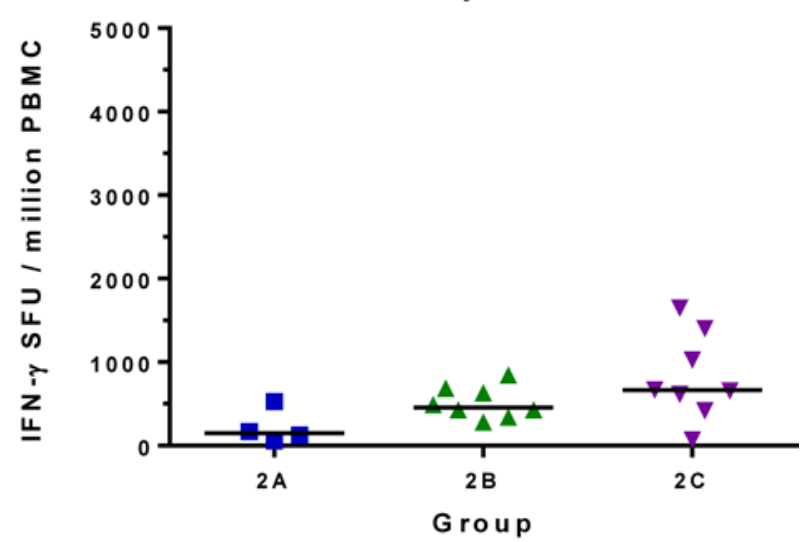

Figure 3. Ex vivo IFN- $\gamma$ T cell response to vaccination. (A) Median ex vivo IFN- $\gamma$ enzyme-linked immunospot (ELISPOT) responses in peripheral blood mononuclear cells (PBMC) to the RH5 insert (summed response across all the individual peptide pools) shown for all groups. Individual responses are shown in Supplemental Figure 1. Median and individual responses are shown at (B) d14 ( $n=4$ vs. 20); (C) d63 (G2A, $n=4$; G2B, $n=8$; G2C, $n=8$ ) assessed by Kruskal-Wallis test with Dunn's multiple comparison test; and (D) d140 (G2A $n=4, \mathrm{G} 2 \mathrm{~B} n=8, \mathrm{G} 2 \mathrm{C} n=8$ ). Symbols are coded according to group. ${ }^{*} P<0.05$, ${ }^{* *} P<0.01$.

The vaccine-induced serum antibody response against RH5_FL as measured by ELISA at d84 was composed of IgG1 and moderate levels of IgG3 (Figure 4D) with little to no IgG2 or IgG4 (Supplemental Figure 5A). Low levels of IgA and IgM were detectable above baseline (d0) levels in only a few volunteers (Supplemental Figure 5A). This profile was maintained at the end of the study period (d140); however, responses were measured at lower levels, consistent with the anti-RH5_FL total IgG ELISA kinetic (Supplemental Figure 5B). The avidity of the anti-RH5_FL IgG, as measured by a sodium thiocyanate displacement (NaSCN displacement) ELISA, was similar at d84 for all volunteers in Groups 2A, 2B, and 2C, with the $\mathrm{IC}_{50}$ (concentration of $\mathrm{NaSCN}$ required to reduce the starting $\mathrm{OD}$ in the ELISA by $50 \%$ ) ranging from 0.8 to $1.7 \mathrm{M}$. Avidity showed a significant increase in Groups $2 \mathrm{~B}$ and $2 \mathrm{C}$ from d28 to $\mathrm{d} 84(n=16, P=$ 0.001 , Wilcoxon matched-pairs signed rank test); however, the same trend was observed in Group 2A ( $n=$ 2), suggesting that this may relate to IgG affinity maturation over time, as opposed to a direct consequence of MVA RH5 boost (Figure 4E).

Previous studies have shown that antibody-secreting cells (ASC) can be detected in peripheral blood for a short time (around d7) after MVA boost when using the ChAd63-MVA regimen (51, 52). RH5_FLspecific ASC responses were assessed by ex vivo ELISPOT using fresh PBMC collected at the d63 visit for volunteers in Groups 2B and 2C. Median responses of 52 vs. 180 RH5_FL-specific ASC per million PBMC were observed, respectively, but there was no significant difference between the 2 groups $(P=0.15$, Mann-Whitney test) (Figure 5A). ASC responses across both groups showed a significant correlation, with peak serum antibody responses at d84 (Figure 5B). 
A

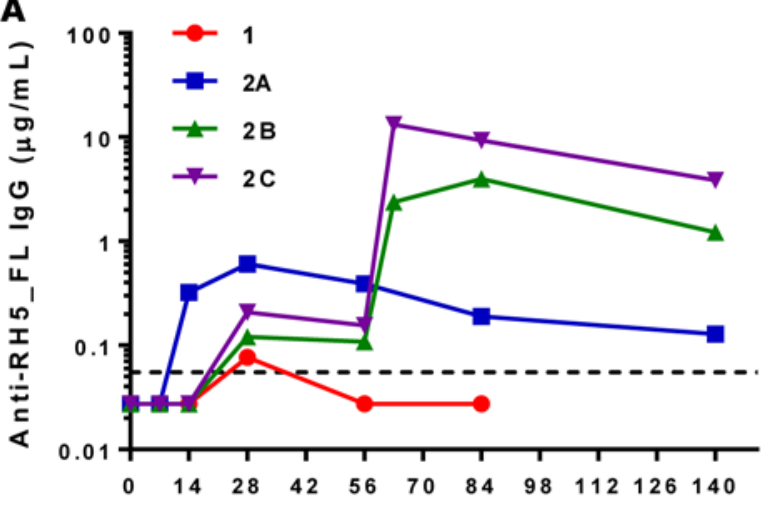

4 Time (d)

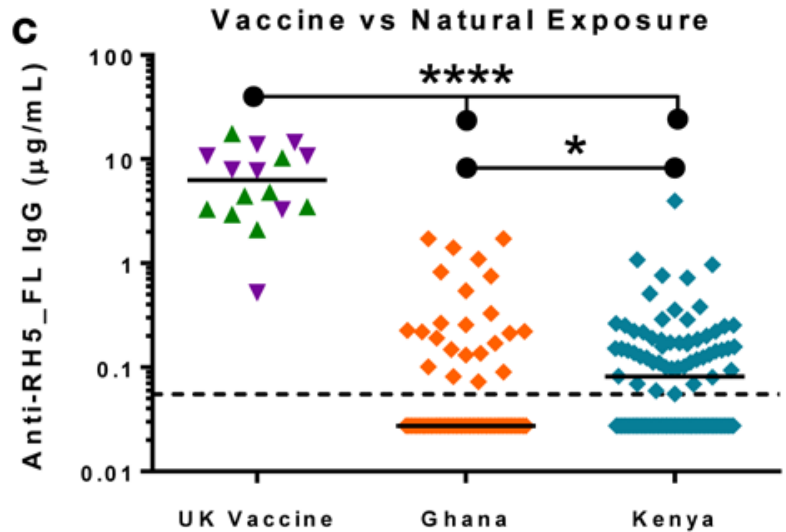

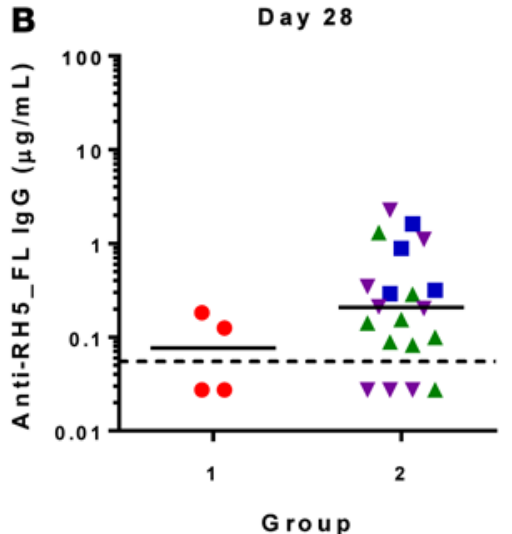

D

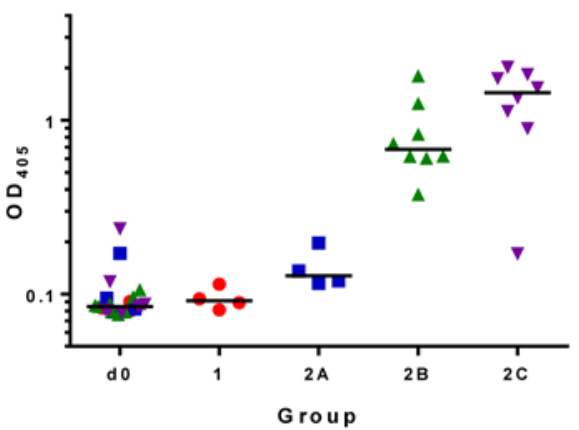

Day 84

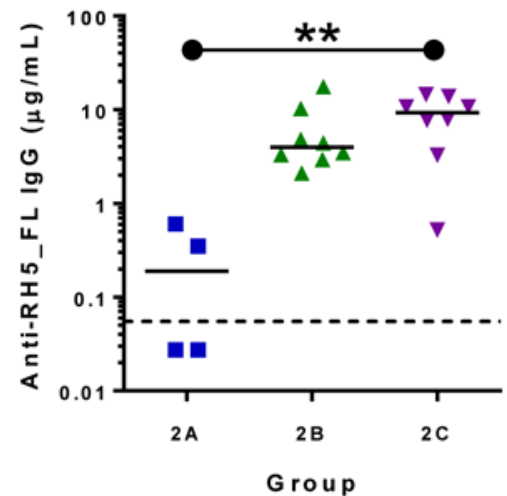

$\lg G 3$

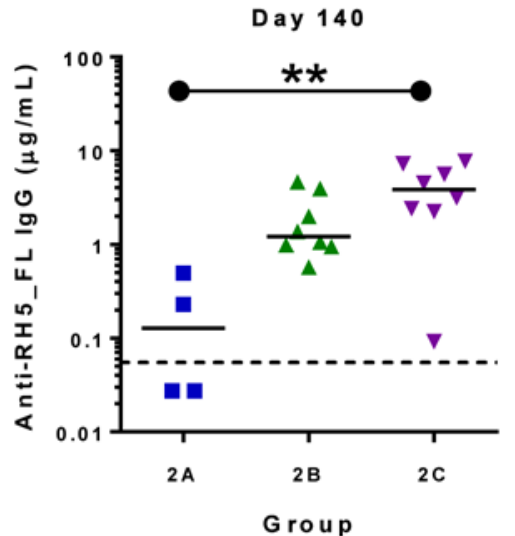

$\mathbf{E}$
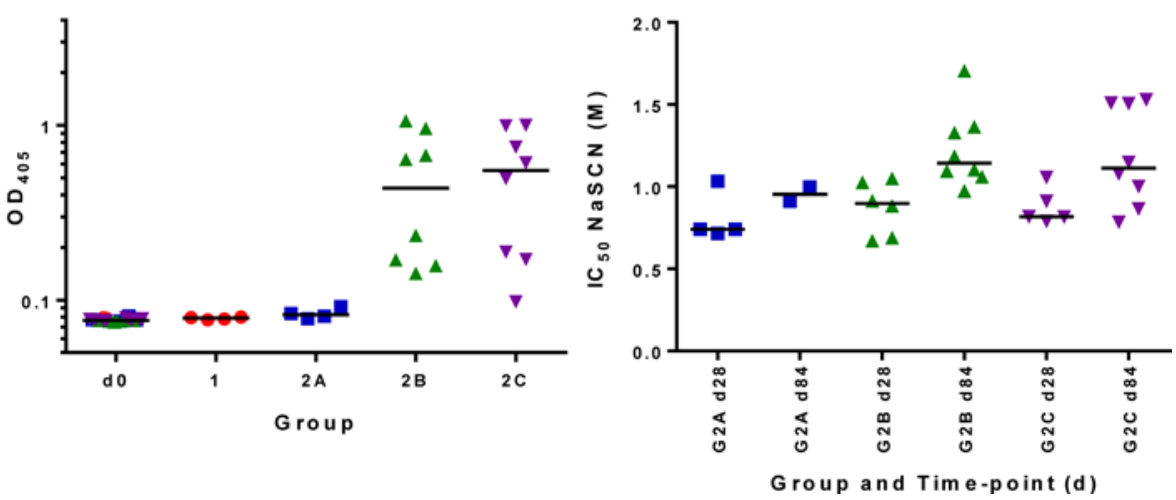

Figure 4. Serum antibody response to vaccination. Group $1(n=4)$, Group $2 \mathrm{~A}(n=4)$, Group $2 \mathrm{~B}(n=8)$, Group $2 \mathrm{C}(n=8)$. (A) Median anti-RH5_FL serum total IgG responses shown for all groups over time. Individual responses are shown in Supplemental Figure 3. Median and individual responses are shown at (B) d28, d84, and d140. The horizontal dotted line indicates the limit of detection of the assay. Statistical analysis using Kruskal-Wallis test with Dunn's multiple comparison test. (C) Vaccine-induced responses shown for Groups 2B and 2C combined $(n=16)$ vs. responses following natural exposure in Ghanaian adults $(n=79)$ and Kenyan adults $(n=96)$; analysis by Kruskal-Wallis test with Dunn's multiple comparison test. (D) Isotype profiles of serum antibody responses against RH5_FL were assessed by ELISA. Responses are shown at baseline (d0) and for all groups at d84. Individual and median responses are shown for IgG1 and IgG3; results for IgG2, IgG4, IgA, and IgM are shown in Supplemental Figure 5. (E) Avidity of serum IgG responses at d28 and d84 was assessed by sodium thiocyanate (NaSCN) displacement RH5_FL ELISA and is reported as the molar (M) concentration of NaSCN required to reduce the starting $\mathrm{OD}$ in the ELISA by $50 \%\left(\mathrm{IC}_{50}\right)$. Only samples with a positive response by anti-RH5_FL total IgC ELISA could be assayed for avidity. Symbols are coded according to group. ${ }^{*} P<0.05,{ }^{* *} P<0.01,{ }^{* * *} P<0.0001$. 
A

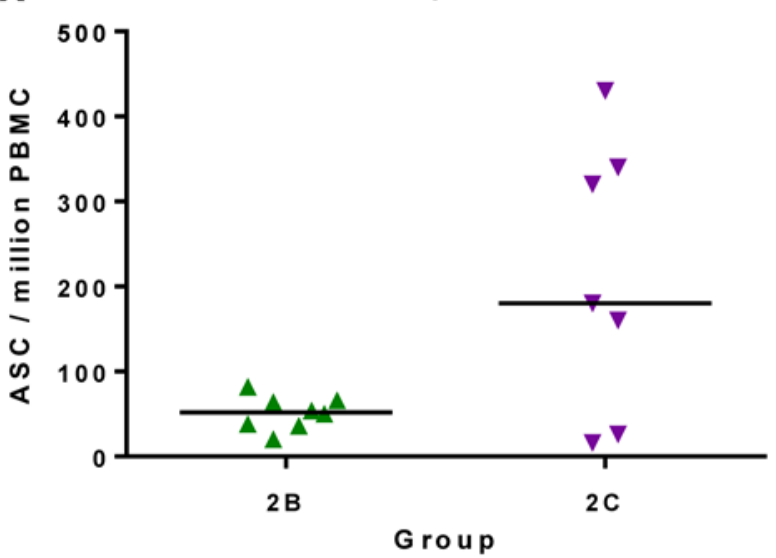

C

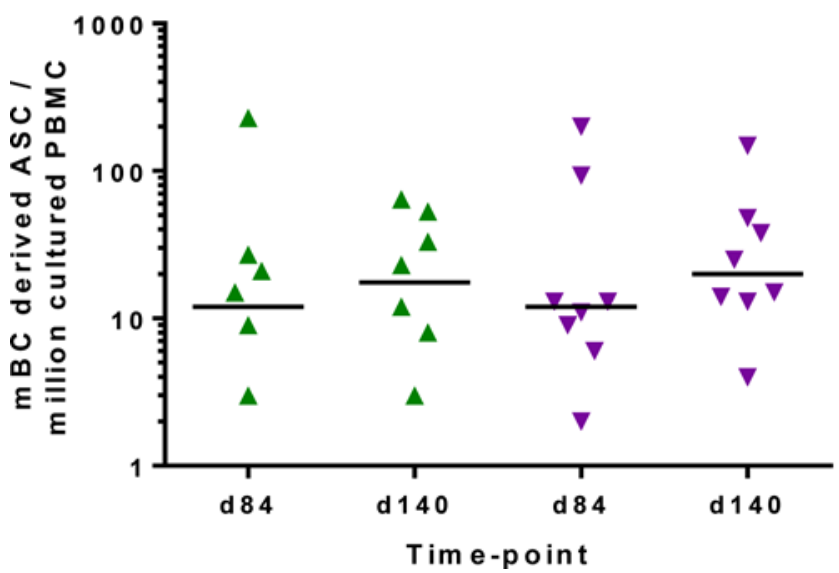

B

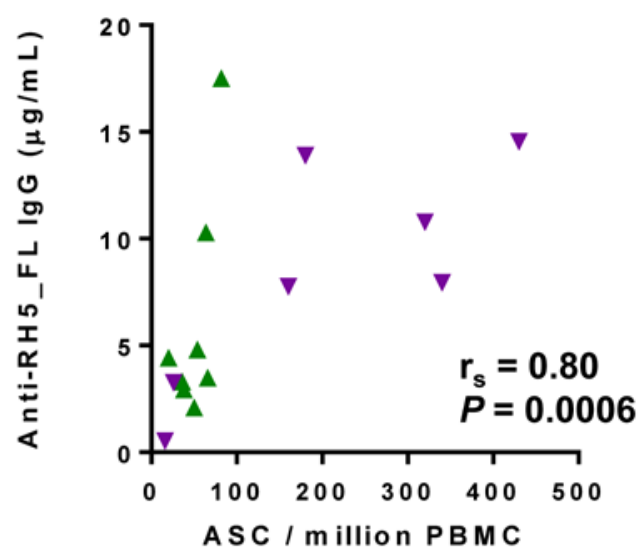

D

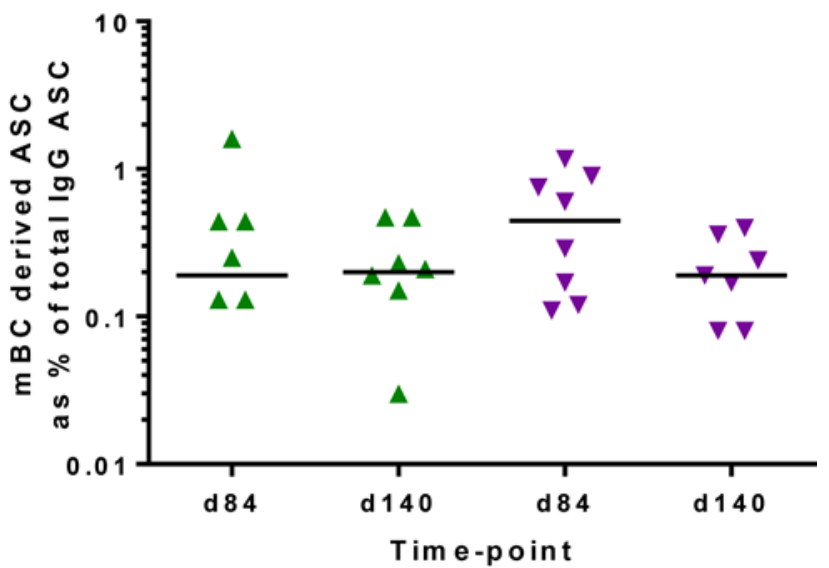

Figure 5. B cell response to vaccination. (A) RH5-specific antibody-secreting cell (ASC) responses were assessed by ex vivo enzyme-linked immunospot (ELISPOT) using RH5_FL protein and fresh peripheral blood mononuclear cells (PBMC) from the d63 time point. Individual and median responses are shown for each group and reported as RH5-specific ASC per million PBMC used in the assay ( $n=8$ for Group 2B and $n=7$ for Group 2C because 1 sample was not tested in this group). (B) Correlation of the ASC response vs. the concentrations of serum anti-RH5_FL IgG measured at d84. Spearman's rank correlation coefficient $\left(r_{s}\right)$ and $P$ value are shown by Spearman's rank correlation. (C) RH5-specific memory B cell (mBC) responses were assessed by ELISPOT assay using RH5_FL protein ( $n=8$ for Groups 2B and 2C). Frozen PBMC were thawed and underwent a 6-day polyclonal restimulation, during which ASC are derived from $\mathrm{mBC}$, before testing in the assay. Individual and median responses are shown from the d84 and 140 time points and are reported as mBC-derived RH5-specific ASC per million cultured PBMC or as (D) \% of total number lgG-secreting ASC ( $n=7$ for Group 2C at the d140 time point in $\mathbf{D}$, otherwise $n=8$ ). Groups $2 \mathrm{~B}$ and $2 \mathrm{C}$ are coded by color and symbol.

$\mathrm{mBC}$ responses were also measured using an established cultured ELISPOT protocol, whereby $\mathrm{mBC}$ within PBMC undergo a 6-day polyclonal stimulation to form ASC, which are then measured using the same protocol as for the ex vivo assay. These were measured for volunteers in Groups $2 \mathrm{~B}$ and $2 \mathrm{C}$ at the d84 and d140 time points (4 and 12 weeks after MVA boost). D84 was most consistently identified as the peak of the $\mathrm{mBC}$ response in other trials of ChAd63-MVA $P$. falciparum blood-stage malaria vaccines (51, 52). Responses are reported as number of mBC-derived RH5-specific ASC per million cultured PBMC (Figure 5C), and as a percentage of total IgG-secreting ASC (Figure 5D). The $\mathrm{d} 84 \mathrm{mBC}$ responses across both groups did not correlate with peak serum antibody responses at $\mathrm{d} 84$ (data not shown). Overall, there were no significant differences between the groups or time points, indicating the $\mathrm{mBC}$ response was well maintained to the end of the study period.

Vaccine-induced antibodies show cross strain in vitro GIA. Serum was analyzed at the GIA Reference Center at NIH, with IgG purified from each sample prior to initial testing against vaccine homologous 3D7 clone parasites. Baseline samples prior to vaccination (d0) using pooled sera, as well as individual samples from Groups 1 and 2A from d84 after vaccination, did not demonstrate any GIA above baseline. Samples from Group 2B and 2C volunteers taken at d84 showed in vitro GIA of median 36.0\% (range $19.7 \%-61.6 \%$ ) and 50.6\% (range 7.2\%-68.1\%) using $10 \mathrm{mg} / \mathrm{ml}$ purified IgG (Figure 6A). We confirmed 

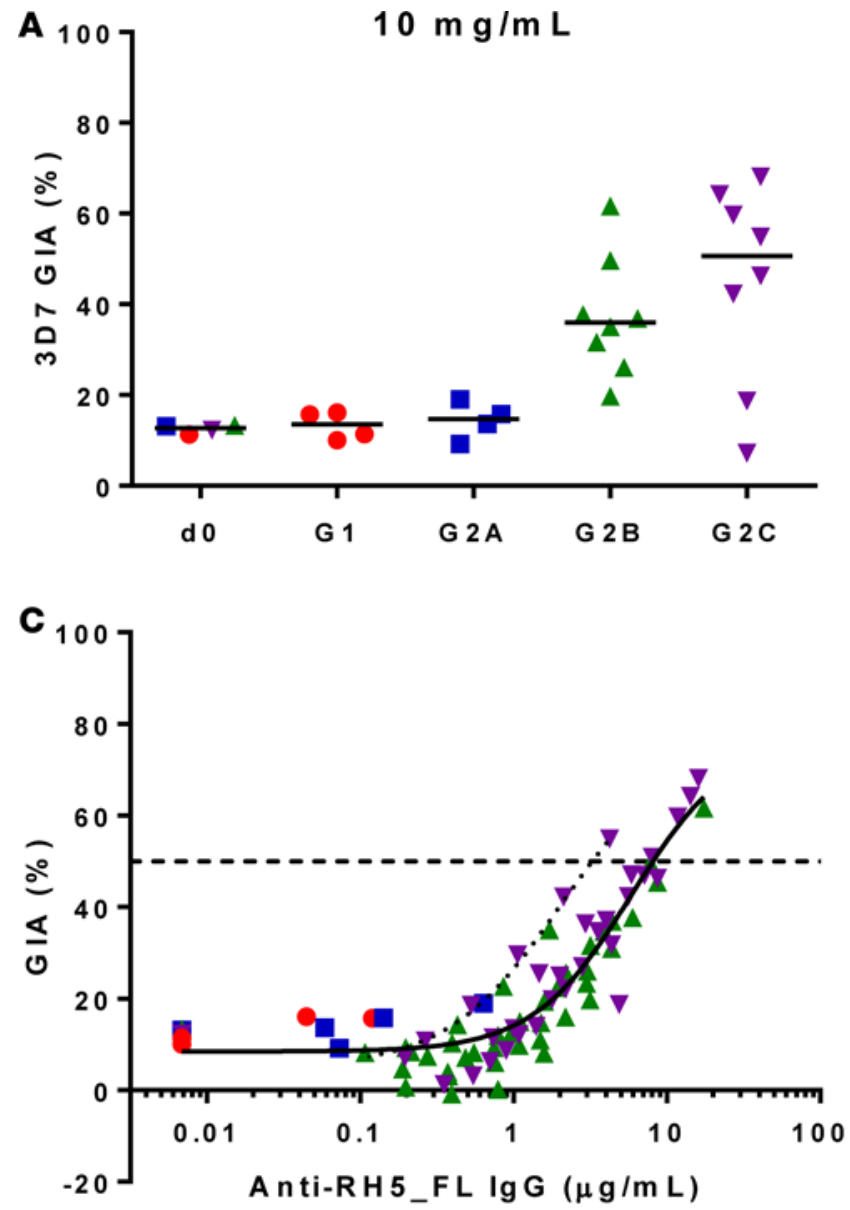

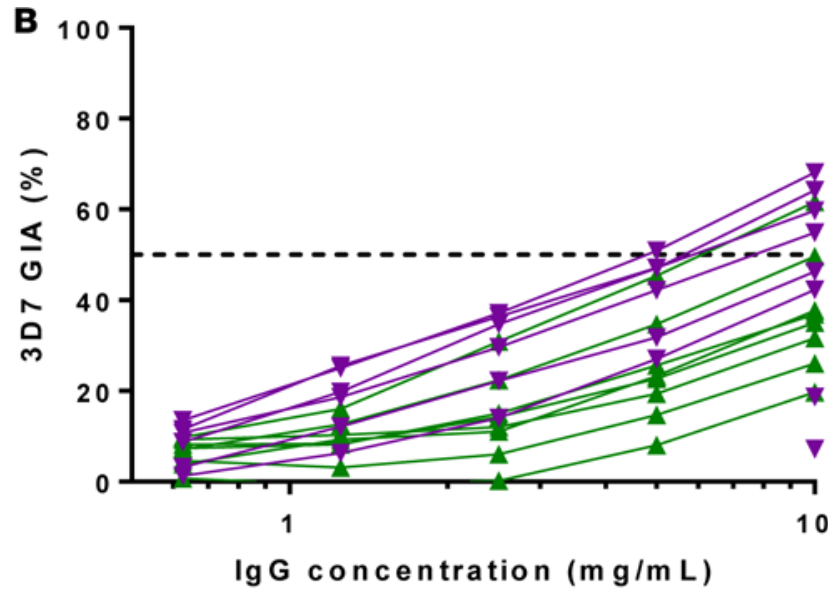

D

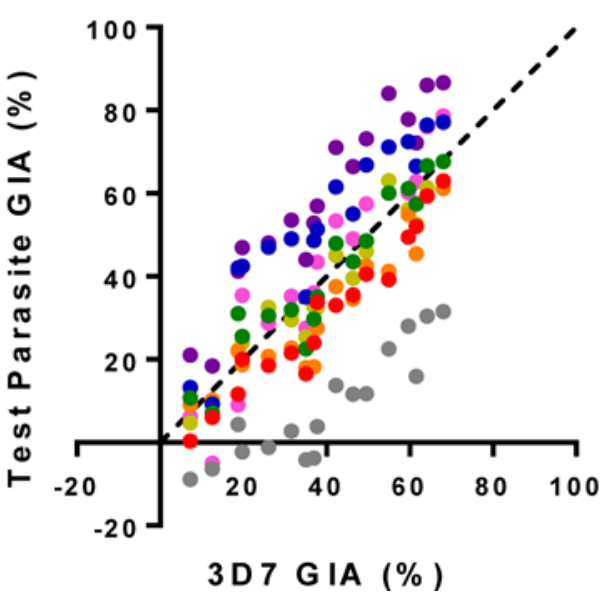

- 7 G 8

- Dd2

- FVO

- G B 4

- MCamp

- HMPOO2

- Cp806

C p 845

Figure 6. Functional GIA induced by ChAd63-MVA RH5 vaccination. (A) In vitro growth inhibition activity (GIA) of purified IgG was assessed at $10 \mathrm{mg} / \mathrm{ml}$ against $3 D 7$ clone $P$. falciparum parasites. Individual data and medians are shown for each group at d84 (G1, $n=4 ; \mathrm{C} 2 \mathrm{~A}, n=4 ; \mathrm{C} 2 \mathrm{~B}, n=8 ; \mathrm{G} 2 \mathrm{C}, n=8$ ); pooled sera were used for each group $(n=4)$ at baseline (d0). (B) Dilution series of purified IgG from Group 2B and 2C d84 samples. (C) Relationship between GIA data from the dilution series shown in $\mathbf{B}$ and concentration of anti-RH5_FL purified IgG used in the assay as measured by ELISA. The EC (concentration $_{50}$ of anti-RH5_FL polyclonal IgC that gives $50 \% \mathrm{GIA}$, dashed line) was $8.2 \mu \mathrm{g} / \mathrm{ml}(95 \% \mathrm{Cl}, 7.2-9.5 \mu \mathrm{g} / \mathrm{ml})$; nonlinear regression curve is shown (solid line, $\mathrm{r}^{2}=$ $0.90, n=74)$. Two volunteers ( 1 in Group $2 \mathrm{~B}$ and $1 \mathrm{in} 2 \mathrm{C}$ ) showed a reproducibly higher $\mathrm{EC}_{50}$ of $3.3 \mu \mathrm{g} / \mathrm{ml}(95 \% \mathrm{Cl}, 2.8-3.9 \mu \mathrm{g} / \mathrm{ml})$; nonlinear regression curve is shown (dotted line, $r^{2}=0.99, n=10$ ). (D) Purified IgG from Group 2B and 2C d84 samples, plus 1 pooled d0 preimmunization sample, were tested at 10 mg/ $\mathrm{ml}$ against a panel of 8 other laboratory-adapted parasite lines and short-term culture-adapted parasite isolates. GIA for each parasite and test sample is plotted against corresponding GIA against $3 D 7$ clone parasites on the $x$ axis.

that GIA decreased as purified IgG was diluted in the assay (Figure 6B) and also that GIA was not enhanced by inclusion of complement (Supplemental Figure 6). The GIA (as routinely assessed, without complement) was related to RH5_FL-specific IgG concentration (Figure 6C), as seen for the merozoite surface protein 1 (MSP1) and apical membrane antigen 1 (AMA1) antigens following human immunization $(9,52,53)$. Notably, the concentration of anti-RH5_FL polyclonal IgG that gives 50\% GIA (EC $\left.{ }_{50}\right)$ was only $8.2 \mu \mathrm{g} / \mathrm{ml}$. Two volunteers also consistently showed a better quality of response, with a 2.5 -fold improvement in the $\mathrm{EC}_{50}(3.3 \mu \mathrm{g} / \mathrm{ml}$ RH5_FL-specific IgG) against the 3D7 clone parasite, although no other obvious differences were apparent in their antibody profile, as measured by the various ELISA assays. This $\mathrm{EC}_{50}$ concentration is substantially lower than previous results with vaccines against the AMA1 and MSP1 antigens (9, 52, 53). In line with this result, the overall levels of GIA using $10 \mathrm{mg} /$ $\mathrm{ml}$ purified IgG from these RH5 vaccinees against 3D7 clone parasites were higher than those achieved with the same ChAd63-MVA platform used previously to deliver AMA1 or MSP1 (54), reaching significance for MSP1 $(P=0.02$, Kruskal-Wallis test with Dunn's multiple comparison test comparing RH5 with AMA1 and MSP1) (Supplemental Figure 7A). However, the levels of GIA using 10 and $2.5 \mathrm{mg} / \mathrm{ml}$ purified IgG against 3D7 clone parasites were comparable with those reported previously in healthy UK adults immunized with an AMA1 recombinant protein vaccine delivered in the proprietary adjuvant sys- 
A
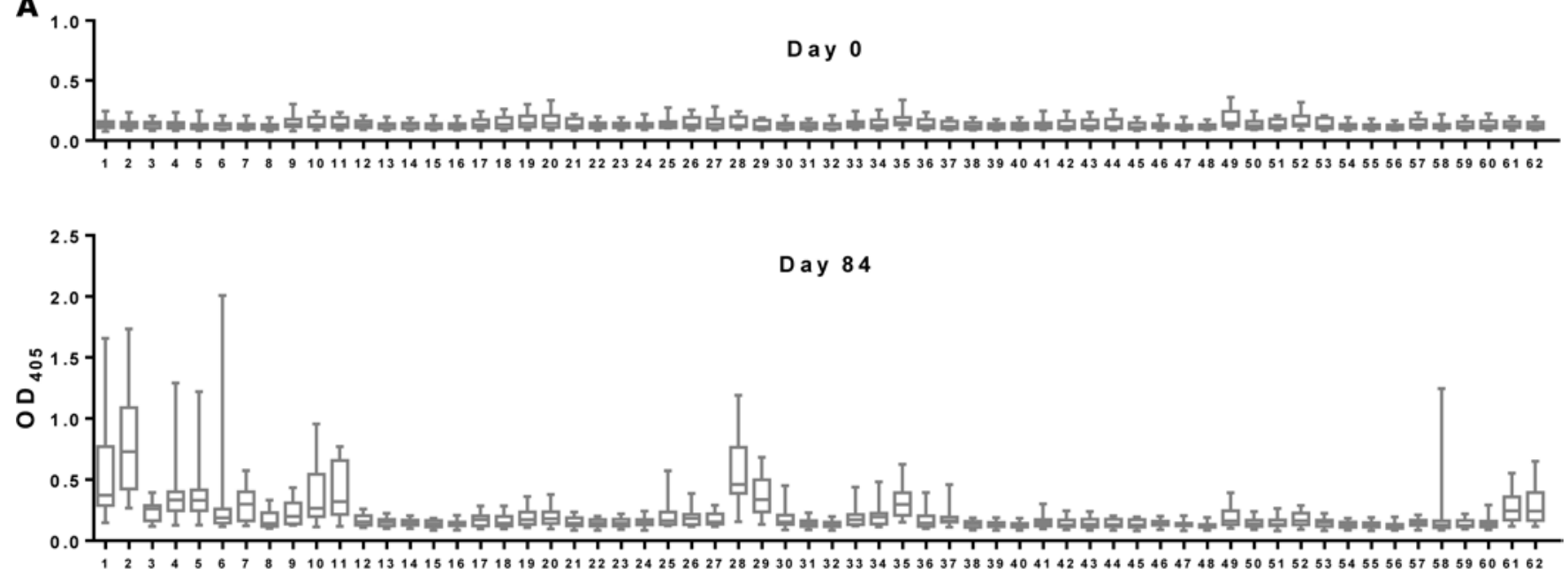

Peptide \#
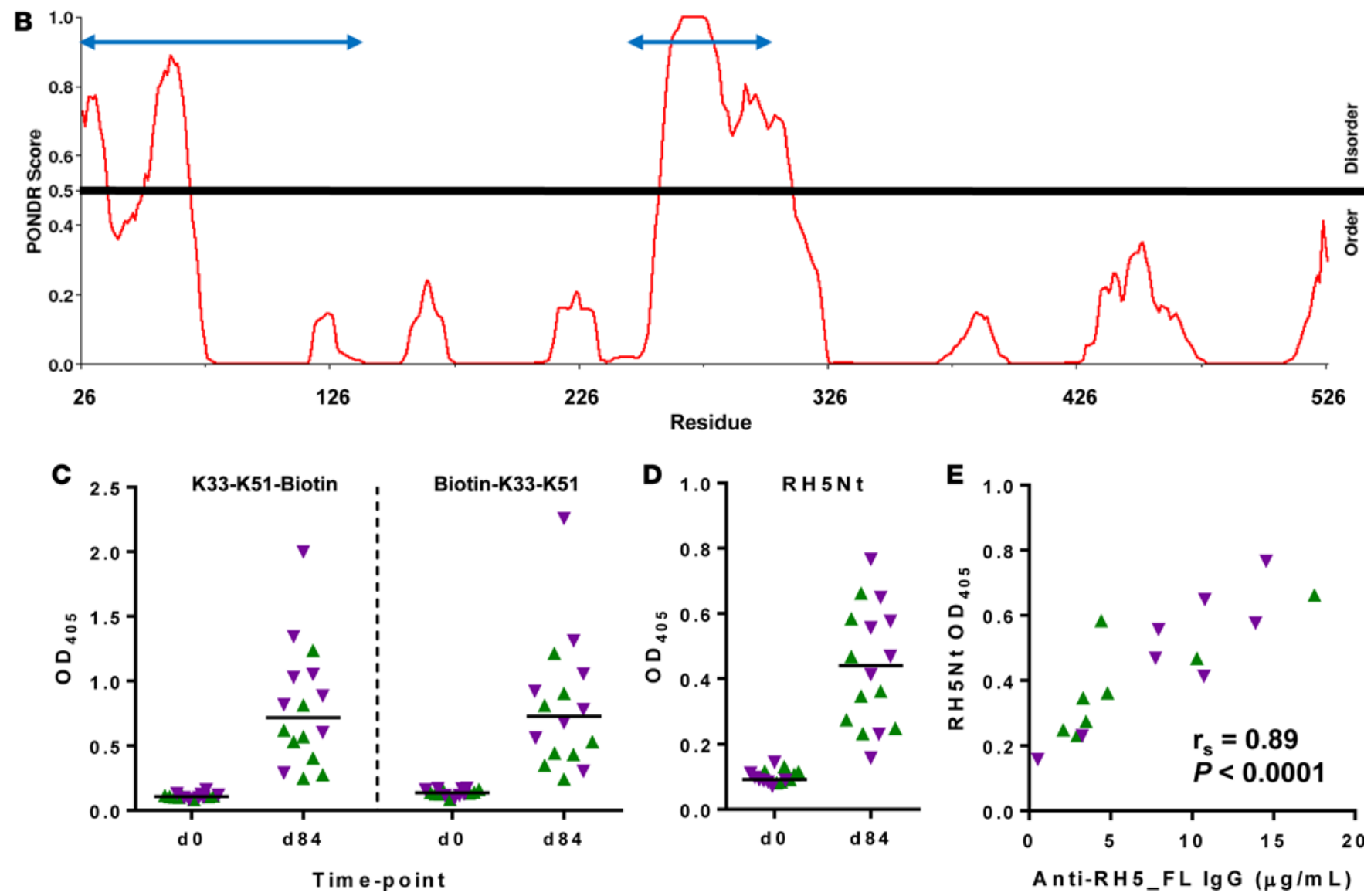

Figure 7. Vaccine-induced anti-RH5 antibodies recognize linear epitopes and RH5Nt. $(\mathbf{A}) \mathrm{DO}$ and d84 sera for volunteers in Groups $2 \mathrm{~B}$ and $2 \mathrm{C}$ ( $n=$ 16) were diluted 1:100 and tested against linear overlapping peptides spanning the RH5 vaccine insert. Median, interquartile range (IQR), and range are shown for each peptide. (B) Plot of disorder within the RH5 vaccine construct predicted by PONDR. Blue arrows indicate the regions removed in the RH5 $\triangle$ NL protein (E26-Y139 and N248-M296). (C) D0 and d84 sera for volunteers in Groups 2B (green triangles) and 2C (purple triangles) ( $n=16$ ) were diluted 1:100 and tested against 19-mer peptides that represent the minimal P113 binding region within RH5Nt (K33-K51). Peptides with N-and C-terminal biotinylation were tested to allow for binding to streptavidin-coated plated in both orientations. Individual and median results are shown. (D) D0 and d84 sera for volunteers in Groups 2B and 2C were diluted 1:100 and tested against RH5Nt protein. Individual and median results are shown $(n=16)$. (E) Correlation of d84 serum IgG responses in Groups 2B and 2C $(n=16)$ against RH5_FL and RH5Nt. Spearman's rank correlation coefficient $\left(r_{s}\right)$ and $P$ value are shown by Spearman's rank correlation. 
tem AS01B from GSK ( $P=0.16$ and $P=0.78$, respectively, Mann-Whitney test) (ref. 53 and Supplemental Figure 7, A and B). This relates to the fact that the AMA1/AS01B formulation was quantitatively 10 times more immunogenic, achieving approximately $100 \mu \mathrm{g} / \mathrm{ml}$ AMA1-specific IgG on average in these vaccinees (53), unlike the $9.3 \mu \mathrm{g} / \mathrm{ml}$ RH5_FL-specific IgG measured on average in Group 2C.

We next assessed the purified IgG from Groups $2 \mathrm{~B}$ and $2 \mathrm{C}$ against a panel of 8 other laboratory-adapted parasite lines (7G8, Dd2, FVO, GB4, MCamp) and short-term culture-adapted parasite isolates (from Cambodian patients with malaria [Cp845, Cp806; ref. 18] or from an Australian resident who contracted malaria in Ghana [HMP002; ref. 55]), that between them include RH5 sequences that encompass the 5 most common RH5 single nucleotide polymorphisms (SNPs) $(18,19)$. All parasites were neutralized by the IgG, with results for each parasite significantly correlating with 3D7 $(n=16, P<0.0001$ and Spearman's rank correlation coefficient $\left[\mathrm{r}_{\mathrm{s}}\right]>0.88$ for all parasites) (Figure 6D). Notably, \%GIA against 1 parasite was significantly lower: Cp806 $(P=0.0001)$; while 2 strains showed significantly higher \%GIA: FVO $(P=0.029)$ and GB4 $(P=$ 0.0009 ) by Friedman test with Dunn's multiple comparison test.

Vaccine-induced antibodies recognize linear and conformational epitopes and inhibit interactions within the RHS complex. We next sought to better understand the fine specificity of the vaccine-induced anti-RH5 antibody response. We initially tested the d84 sera from all volunteers in Groups 2B and 2C by ELISA against a linear peptide array spanning the RH5_FL vaccine sequence (Supplemental Table 3 and Figure 7A). Responses were detected to the N-terminal region, as well as a central region and a small part of the C-terminus, suggesting the vaccine-induced anti-RH5_FL IgG do recognize linear epitopes within the RH5 molecule. Interestingly, these responses to linear epitopes mainly correspond to predicted regions of disorder within the RH5 molecule (Figure 7B). Further analysis of the peptide data highlighted peptide 28 - against which the sera from all 16 volunteers reacted - which is also recognized by the nonneutralizing mouse mAb 4BA7 (56), as well as peptide 5 in RH5Nt, which is recognized by the nonneutralizing mouse mAb RB3 (56). Other peptides of interest include peptides 1-3 within RH5Nt, given that the P113-binding region was previously mapped to a 19-mer sequence running from aa K33-K51 (29). These peptide reagents included the N38Q aa substitution in the vaccine construct to remove a site of potential N-linked glycosylation. We therefore synthesized the native 19-mer sequence, biotinylated at either the $\mathrm{N}$ - or the C-terminus (Supplemental Table 3), and assessed responses by ELISA (Figure 7C). Similar to the results with the original peptide 2, all volunteers showed detectable responses irrespective of bound peptide orientation. We also confirmed these results using recombinant RH5Nt protein in ELISA (Figure 7D), and these data would suggest the sera have the potential to inhibit the RH5Nt-P113 interaction. Notably, these responses to RH5Nt significantly correlated with those against RH5_FL (Figure 7E), suggesting that in each vaccinee, the response to RH5Nt is a consistent proportion of the total anti-RH5_FL response irrespective of overall magnitude.

To assess whether responses are also directed against conformational epitopes, we next performed ELISA against heat-denatured RH5_FL protein (Figure 8A). These data showed significant loss of sero-reactivity in all vaccinees $(P=0.008$ for both groups by Wilcoxon matched-pairs signed rank test) - similar to the conformation-sensitive mAb $2 \mathrm{AC} 7(49,56)$, but not mAb 4BA7, which binds a linear peptide (56). To investigate further, we performed ELISA using RH5 $\Delta$ NL protein, which lacks the disordered N-terminal and central loop regions of RH5 (49), and quantified these responses by CFCA. Responses to this protein were concordant with those against RH5_FL (Figure 8B), suggesting that most of the vaccine-induced IgG recognize the RH5 $\Delta$ NL structured protein and that the linear peptide array and RH5Nt ELISA are measuring only a minor proportion of the total anti-RH5_FL response.

We next assayed the ability of these sera to inhibit interactions within the RH5 complex by avidity-based extracellular protein interaction screen (AVEXIS). These data confirmed that the d84 sera from the Groups 2B and 2C volunteers could inhibit the interaction between RH5 and P113, as well as RH5 and basigin and RH5 and CyRPA (Figure 9A). One volunteer in Group 2C showed no inhibition, consistent with their very low postboost anti-RH5_FL IgG concentration (Supplemental Figure 3D). Notably, the blocking activity observed by AVEXIS for each of the 3 interactions significantly correlated with the antiRH5_FL IgG response (Figure 9B).

\section{Discussion}

This phase Ia dose escalation and safety clinical trial reports the first data to our knowledge in humans for a vaccine targeting the RH5 antigen from the blood-stage $P$. falciparum merozoite. We show in healthy malaria-naive UK adult volunteers that a recombinant ChAd63-MVA heterologous prime-boost immu- 
A

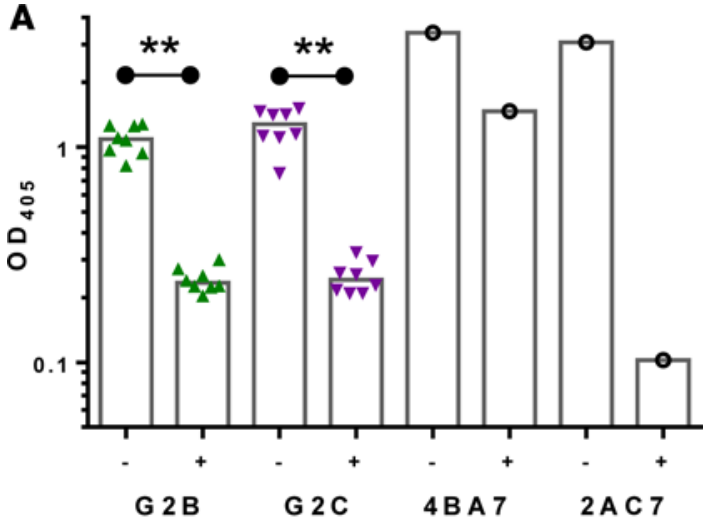

B

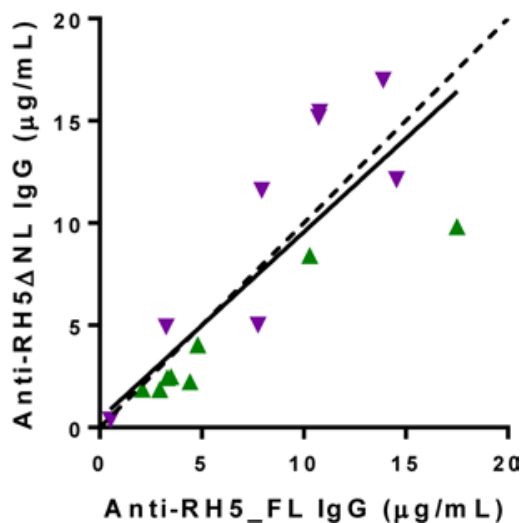

Figure 8. Vaccine-induced antibodies recognize conformational epitopes and inhibit interactions within the RH5 invasion complex. (A) DO and d84 sera for volunteers in Groups $2 B$ and $2 C(n=16)$ were tested by ELISA against nondenatured RH5_FL protein (-) and the same protein following heat denaturation (+). Individual and median responses are shown. The 4BA7 and 2AC7 mAbs were included as controls that bind a linear vs. conformational epitope, respectively. ${ }^{* *} P<0.01$ according to Wilcoxon matched-pairs signed rank test. (B) D84 serum ELISA responses to RH5_FL and RH5 $\triangle \mathrm{NL}$ for volunteers in Croups $2 \mathrm{~B}$ and $2 \mathrm{C}(n=16)$ were analyzed for concordance by linear regression (solid line). $\mathrm{r}^{2}=$ 0.69; slope $=0.91(95 \% \mathrm{Cl}, 0.56-1.27) ; \mathrm{Y}$ intercept when $\mathrm{X}=0.0$ is $0.4(95 \% \mathrm{Cl},-2.7-3.6)$; $\mathrm{X}$ intercept when $\mathrm{Y}=0.0$ is -0.5 $(95 \% \mathrm{Cl},-6.0-2.3)$. Line of identity $(\mathrm{X}=\mathrm{Y})$ is also shown (dashed line).

nization regimen has a favorable safety profile and can induce functional RH5-specific-serum antibody responses, in addition to B and T cell responses. Reactogenicity of the ChAd63 RH5 vector was similar to that seen with the same doses of ChAd63 vectored vaccines encoding the $P$. falciparum antigen multiepitope string-thrombospondin-related adhesion protein (ME-TRAP), circumsporozoite protein (CSP), MSP1, or AMA1 $(36-39,52,54)$ or the P. vivax antigen Duffy-binding protein region II (PvDBP_RII) (43). The same vectors encoding ME-TRAP have similarly been safe following immunization of adults, children, and infants residing in malaria-endemic areas $(41,42)$. Our data with ChAd63 RH5 add to the growing body of evidence that this simian adenovirus vector is safe for clinical use. Likewise, the clinical safety of MVA as a recombinant vaccine vector for many infectious diseases and cancer is now well documented (57). MVA RH5 appeared to be more reactogenic than the ChAd63 vector at the higher dose, consistent with previous vaccine trials using this orthopoxvirus vector $(38,39,43,44,46,58)$.

The ChAd63 and MVA vectors used here were designed to maximize induction of antibody responses against blood-stage malaria antigens, in conjunction with the $\mathrm{T}$ cell responses for which they are more routinely used $(32,33)$. In this trial, RH5-specific IFN- $\gamma \mathrm{T}$ cell responses were induced and peaked following the MVA boost at median levels of $>2,000 \mathrm{SFU} /$ million PBMC. No data on cellular responses to this antigen have been reported following natural $P$. falciparum infection; however, given that vaccination elicited peptide-restimulated responses spanning the entire RH5 sequence, the RH5_FL molecule does not appear to lack $\mathrm{T}$ cell epitopes. The kinetics and magnitude of the response were also similar to those previously reported following human vaccination with the same vectors encoding $P$. falciparum or $P$. vivax antigens $(36-39,43)$. Similarly, studies using chimpanzee adenovirus vectors followed by MVA boost $(44,46,58)$ have routinely shown that a mixed antigen-specific $\mathrm{CD} 4^{+} / \mathrm{CD} 8^{+} \mathrm{T}$ cell response is induced in humans. The possible contribution of $\mathrm{T}$ cells to blood-stage malaria protection remains unclear, given that previous clinical trials using whole parasite immunization (59) or ChAd63-MVA vectors encoding MSP1 or AMA1 (54) failed to show an impact on blood-stage parasite growth following controlled human malaria infection (CHMI) with $P$. falciparum despite strong $\mathrm{T}$ cell induction by vaccination. However, $\mathrm{CD} 8^{+} \mathrm{T}$ cells against blood-stage antigens can act against late liver-stage forms of the parasite once merozoite antigens are expressed $(54,60)$, while viral vector vaccine-induced $\mathrm{CD} 4^{+} \mathrm{T}$ cells will provide key help to $\mathrm{B}$ cell responses $(61,62)$.

In agreement with preclinical data in mice, rabbits, and Aotus monkeys $(12,34)$, the ChAd63-MVA RH5 vaccines induced RH5_FL-specific serum IgG antibody responses, peaking at a median of $0.2 \mu \mathrm{g}$ / $\mathrm{ml}$ after ChAd63 prime and $9.3 \mu \mathrm{g} / \mathrm{ml}$ after MVA boost in the full-dose vaccination Group $2 \mathrm{C}$. The overall kinetic and magnitude of the RH5_FL-specific IgG, ASC, and $\mathrm{mBC}$ responses induced here in malaria-naive humans are broadly comparable with those reported for the same vectors encoding PvDBP_RII (43) or P. falciparum MSP1 and AMA1 $(38,39,51,52)$. These vaccine-induced RH5_FL-specific IgG concentra- 
A

G2B d0

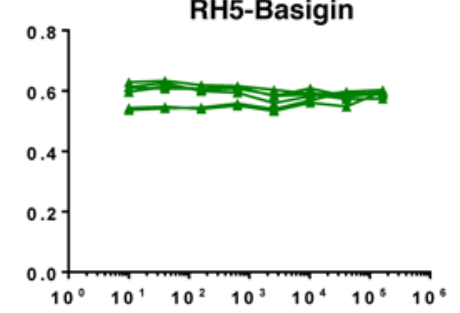

G2B d84

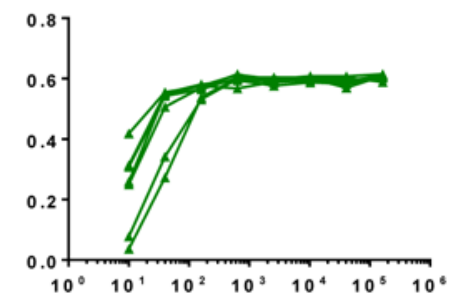

G2C do

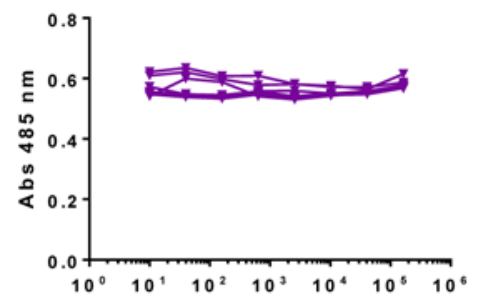

G2C d84

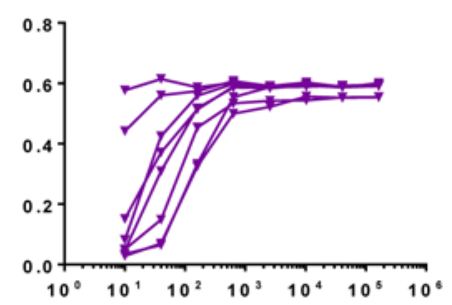

Control

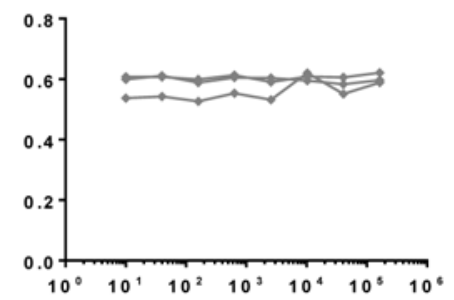

RH5-CyRPA
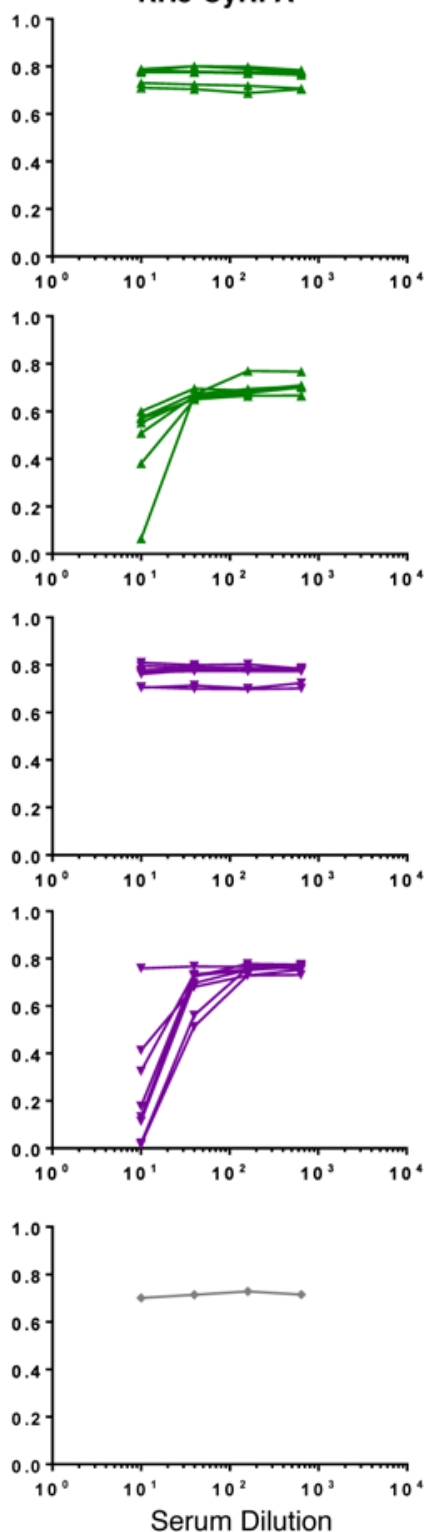

RH5-P113
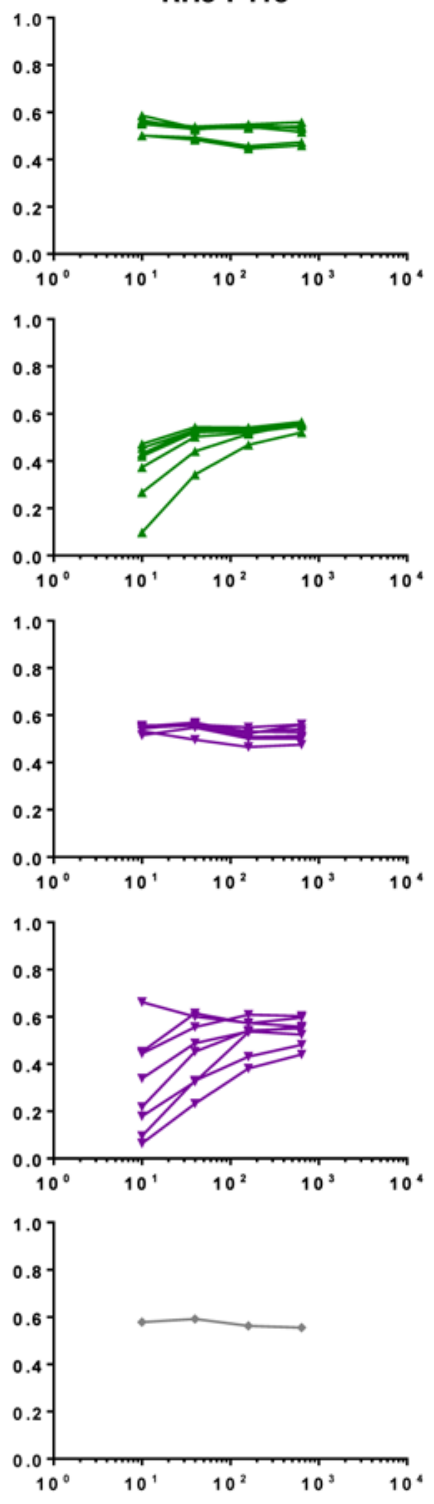

B
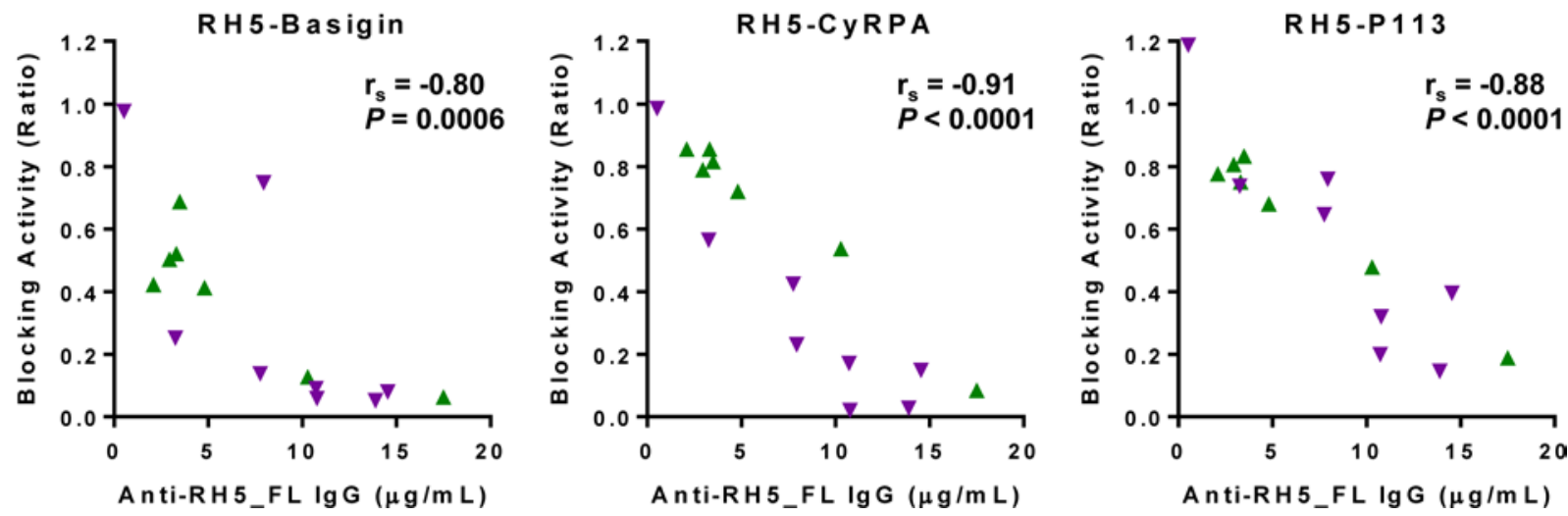

Figure 9. Vaccine-induced antibodies inhibit interactions within the RH5 invasion complex. (A) DO and d84 Group 2B sera ( $n=7)$ and Group $2 \mathrm{C}$ sera $(n=8)$ were tested for their ability to inhibit the interaction between proteins from the RH5 invasion complex by AVEXIS. Dilution of each test serum sample is shown starting at 1:10. Results with various assay controls also shown (no serum for RH5-Basigin, and anti-AMA1 for RH5-CyRPA and RH5-P113). Each point represents the mean of duplicate or triplicate wells. (B) Correlation of blocking activity for each interaction using d84 
sera from Groups 2B and 2C ( $n=15)$ against anti-RH5_FL serum IgG responses measured by ELISA. Blocking activity was calculated for each individual sample from the data in panel $\mathbf{A}$ as the ratio of the $A b s 485 \mathrm{~nm}$ at 1:10 serum dilution divided by the Abs $485 \mathrm{~nm}$ at the highest serum dilution tested. Spearman's rank correlation coefficient $\left(r_{s}\right)$ and $P$ value(Spearman's rank correlation) are shown.

tions are similar to those observed following ChAd63-MVA immunization with PvDBP_RII (43) but are lower than P. falciparum AMA1 $(38,52)$ and MSP1 (38) and higher than CSP (37). Notably, in agreement with immunoepidemiological datasets $(12,23-26)$, anti-RH5_FL IgG responses were weak in the Kenyan and Ghanaian adult populations assessed here for comparison, with median responses $<100 \mathrm{ng} / \mathrm{ml}$. The vaccine data, thus, confirm that RH5 is not in itself a weakly immunogenic antigen in humans but instead suffers modest immunogenicity in the context of natural $P$. falciparum infection.

Consistent with other ChAd63-MVA malaria vaccines $(43,52,63)$, the anti-RH5_FL serum IgG response was largely composed of IgG1 and IgG3, with moderate avidity as measured by NaSCN displacement ELISA. A study of naturally exposed individuals in Mali reported that the predominant IgG isotype detected against RH5 was IgG3 (23). A similar result was observed in children from Papua New Guinea (64), suggesting qualitative aspects of the vaccine-induced response may differ to those induced by natural exposure. Overall, the relevance of antibody isotype, affinity, and avidity to in vivo protection in humans against the $P$. falciparum merozoite remain debated, although the study in Papua New Guinea associated the IgG3 response against RH5 with reduced risk of malaria (64).

We subsequently assessed the functional anti-parasitic antibody activity using the standardized in vitro assay of GIA. In agreement with preclinical studies, these data confirmed the vaccine-induced antibodies in humans were strain transcending, showing activity against all tested parasites. Notably, addition of human complement did not increase levels of GIA, as reported for some merozoite surface proteins (65). This is perhaps not surprising, given that antibodies only have a very short window of opportunity to bind RH5 following its release from the rhoptries, likely leading to time constraints on complement recruitment. Notably, some individual parasite laboratory lines or short-term culture-adapted parasite isolates significantly differed in their sensitivity to neutralization in comparison with the 3D7 reference clone - with 2 being easier to neutralize and 1 less so. The reasons for these differences remain to be investigated. The panel of parasites used here covered a significant breadth of the known RH5 sequence diversity $(18,19,21)$, encompassing at least 8 catalogued nonsynonymous SNPs, including the top fifth and seventh most common SNPs by global minor allele frequency (MAF) at positions 148, 147, 197, 203, 410, and 407 (MalariaGEN v4.0, ref. 66). A previous rabbit study using the same parasite isolates from Cambodian patients showed a similar range of susceptibility to vaccine-induced anti-RH5_FL IgG (18), which was not associated with the presence of small numbers of specific polymorphisms. It remains likely that other aspects of the RH5 invasion complex biology can affect the susceptibility of individual parasite isolates to anti-RH5 antibodies.

Most encouragingly, the GIA $\mathrm{EC}_{50}$ concentration of RH5_FL-specific polyclonal human IgG against 3D7 clone parasites was only $8 \mu \mathrm{g} / \mathrm{ml}$. A previous study affinity-purified RH5-specific human IgG from plasma of naturally exposed individuals in Mali and tested for GIA, reporting an $\mathrm{EC}_{50}$ concentration of $55 \mu \mathrm{g} /$ $\mathrm{ml}$ (23), which suggests the vaccine-induced IgG here may be qualitatively superior to the responses induced against RH5 following natural parasite exposure. Moreover, this level is substantially lower than previous results with vaccines tested in humans against the AMA1 and MSP1 antigens, where GIA EC ${ }_{50}$ concentrations were reported from $70-100 \mu \mathrm{g} / \mathrm{ml}(9,52,53)$ and $>600 \mu \mathrm{g} / \mathrm{ml}$ (9) for AMA1- and MSP1-specific human IgG, respectively. Consequently, the levels of GIA observed here with ChAd63-MVA RH5 outperformed those previously observed in humans with the same vectors encoding MSP1 or AMA1 (54), given that these vaccines all induce quantitatively similar levels of IgG. Our data, thus, identify a clear hierarchy, whereby the RH5 antigen is inherently more susceptible to vaccine-induced human IgG than the historically favored AMA1 and MSP1 target antigens - consistent with preclinical data in rabbits (18) and in vivo protection data in Aotus monkeys (34), boding well for future efficacy testing of RH5-based vaccines.

Finally, we further sought to better understand the breadth of epitopes recognized by anti-RH5_FL IgG. We initially identified regions of linear antibody epitopes within the RH5_FL molecule by peptide array, including the disordered N-terminus and internal loop between helices 2 and 3 (49). Mouse mAbs that bind linear peptides within both of these regions have been shown to be nonneutralizing (56); however, immunization data with RH5Nt (29) and another mAb study (67) suggest the N-terminal region of the molecule can elicit functional antibodies that may block P113 binding and/or interfere with proteolytic pro- 
cessing of RH5_FL. Further analysis confirmed the vaccine-induced IgG also recognized conformational epitopes. Quantitative assessment by ELISA and CFCA using the structured RH5 NL protein (which lacks the disordered $\mathrm{N}$-terminus and internal loop) suggested that most of vaccine-induced IgG recognize this region of RH5 and that antibodies against RH5Nt constitute only a minor, but consistent, proportion of the total anti-RH5_FL response. Subsequent testing by AVEXIS confirmed the sera from vaccinees could block the interaction of RH5_FL with P113, CyRPA, and basigin, consistent with known information relating to the binding of these molecules $(29,49,56,68)$ and the measurable antibody responses against both RH5Nt and RH5 $\triangle \mathrm{NL}$. Interestingly, blocking activity for all 3 interactions correlated with the anti-RH5_FL IgG response, suggesting qualitatively similar responses were induced in all vaccinees. Our ongoing work will focus on elucidating epitopes recognized by vaccine-induced human B cells in order to guide future immunomonitoring and better understand mechanisms of $P$. falciparum merozoite neutralization and inhibition of these interactions within the complex.

Overall, substantial progress has been made since RH5_FL was first reported as a promising new vaccine candidate in late 2011. Here, we demonstrate for the first time to our knowledge, using a rapidly translated viral vectored vaccine platform, that substantial RH5_FL-specific antibodies and B cell and T cell responses can be induced safely by immunization in humans, in contrast to the levels of responses observed following years of natural malaria exposure. Our recent demonstration of a blood-stage CHMI model for vaccine testing using $P$. falciparum (53) will allow for rapid phase IIa proof-of-concept efficacy testing of RH5-based vaccines in the near future.

\section{Methods}

Detailed methods are provided in Supplemental Methods.

ChAd63 and MVA RH5 vaccines. The design, production, and preclinical testing of the viral vector vaccines have been reported previously in detail $(12,34)$. Briefly, both recombinant viruses express the same $1,503 \mathrm{bp}$ coding sequence of RH5 from the 3D7 clone of $P$. falciparum, aa E26-Q526 (NCBI accession number XM_001351508.1). ChAd63 RH5 was manufactured by Advent, which is a daughter company of ReiThera. This production facility meets current Good Manufacturing Practice (cGMP) requirements of the US Food and Drug Administration (FDA) and the European Medicines Agency (EMA) to produce investigational vaccines to be used in human clinical studies. MVA RH5 was manufactured under cGMP conditions by IDT Biologika GmbH, Germany, as previously described (38).

Study design. VAC057 was a first-in-human, open-label, nonrandomized, dose-escalation phase Ia clinical trial evaluating the safety and immunogenicity of the viral vectored vaccines ChAd63 RH5 and MVA RH5 in a heterologous prime-boost regime with an 8-week interval. The study was conducted in the UK at the Centre for Clinical Vaccinology and Tropical Medicine (CCVTM; University of Oxford, Oxford, United Kingdom) and the NIHR Wellcome Trust Clinical Research Facility (WTCRF) in Southampton, UK. The trial was registered on Clinicaltrials.gov (NCT02181088). The primary endpoint of the study was to assess the safety of ChAd63 RH5 and MVA RH5, with a secondary endpoint to assess immunogenicity.

Participants. Healthy, malaria-naive males and nonpregnant females aged 18-50 were invited to participate in the study. Volunteers were recruited and vaccinated at the CCVTM, part of the Oxford Vaccine Centre (OVC), at the University of Oxford and the NIHR WTCRF in Southampton. Twenty-four volunteers were enrolled in total. A full list of inclusion and exclusion criteria is reported in Supplemental Methods.

Safety analysis. Following each vaccination, volunteers completed an electronic diary card for 28 days with any AE data. AE data were also collected at follow-up visits. Observations (heart rate, temperature, and blood measures) were taken at the clinic visits from the day of vaccination until the $\mathrm{d} 28$ follow-up visit. Blood tests for exploratory immunology were taken at all visits except those occurring 2 days after each vaccination (i.e., d2 and d58). Blood samples for safety (full blood count, liver function, urea, and electrolytes) were carried out at screening, d0, d7, and d28 for all groups, as well as on d56, d63, and d84 for Groups 2B and $2 \mathrm{C}$. Any solicited AEs occurring during the diary card period were defined as being at least possibly related to vaccination. The likely causality of all other AEs was assessed as described in the protocol, and all AEs considered possibly, probably, or definitely related to vaccination are reported (Supplemental Table 1). Further details on grading are provided in the Supplemental Material.

Peptides. Peptides for ex vivo IFN- $\gamma$ ELISPOT and the K33-K51 ELISA peptides were purchased from NEO Scientific (Supplemental Table 2), while the biotinylated overlapping 20-mer peptides for ELISA were synthesized by Mimotopes (Supplemental Table 3). 
Recombinant RH5 proteins for ELISAs and B cell assays. Recombinant RH5_FL and RH5 were generated using Drosophila melanogaster Schneider 2 (S2) polyclonal stable cell lines (ExpreS ${ }^{2}$ platform, ExpreS²ion Biotechnologies) (69), while RH5Nt protein was produced using HEK293-6E cells as previously described (29).

Ex vivo IFN- $\gamma$ ELISPOT. Ex vivo IFN- $\gamma$ ELISPOT was used to assess the kinetics and magnitude of the vaccine-induced $\mathrm{T}$ cell responses over time. Fresh PBMC were used in all assays using a previously described protocol (39). Results are expressed as IFN- $\gamma$ SFU per million PBMC.

Total IgG ELISAs. ELISAs were performed using standardized methodology as previously described $(38,39)$, except that plates were coated with recombinant RH5_FL or RH5 $\Delta$ NL protein produced from the Drosophila S2 cells. Responses measured in AU are reported in $\mu \mathrm{g} / \mathrm{ml}$ following generation of a conversion factor by calibration-free concentration analysis (CFCA).

Avidity and isotype ELISAs. IgG antibody avidity was assessed by NaSCN displacement ELISA using previously described methodology (63), except that plates were coated with recombinant RH5_FL produced from the Drosophila S2 cells at $2 \mu \mathrm{g} / \mathrm{ml}$. The concentration of NaSCN required to reduce the $\mathrm{OD}_{405}$ (optical density measured at $405 \mathrm{~nm}$ )to $50 \%$ of that without NaSCN was used as a measure of avidity $\left(\mathrm{IC}_{50}\right)$. Antibody isotype ELISAs were also performed using methodology described in detail elsewhere (63) with the same exception as for the avidity ELISA.

$m B C$ and ASC ELISPOT. mBC ELISPOT assays using RH5_FL protein were performed as described in detail elsewhere (51). Ex vivo ASC ELISPOT assays were performed using fresh PBMC directly prepared and added to the ELISPOT plate with no preceding 6-day culture.

Assay of GIA. The ability of antibodies to inhibit growth of $P$. falciparum parasites in vitro was assessed at the GIA Reference Center (NIAID, NIH) as previously described (70). All samples were tested at $10 \mathrm{mg} /$ $\mathrm{ml}$ in a final test well, followed by a dilution series for positive samples to determine the concentration that gave $50 \%$ GIA $\left(\mathrm{EC}_{50}\right)$. Serum IgG concentrations were measured using HPLC.

AVEXIS. AVEXIS was performed essentially as described (71) using d0 and d84 serum. Controls included addition of no serum or addition of polyclonal rabbit IgG raised against AMA1 (19).

Statistics. Data were analyzed using GraphPad Prism version 6.07 for Windows (GraphPad Software Inc.). All tests used were 2-tailed and are described in the text. A value of $P<0.05$ was considered significant.

Study approval. The VAC057 study received ethical approval from the Oxfordshire Research Ethics Committee A in the UK (REC reference 14/SC/0120). The study was also reviewed and approved by the UK Medicines and Healthcare products Regulatory Agency (MHRA, reference 21584/0331/001-0001). Volunteers signed written consent forms, and consent was verified before each vaccination. The trial was conducted according to the principles of the current revision of the Declaration of Helsinki 2008 and in full conformity with the ICH guidelines for Good Clinical Practice (GCP).

\section{Author contributions}

ROP, SES, SCE, KM, AD, FG, HDG, NJB, IDP, OJG, NJE, JJ, GML, DGWA, LS, SDM, NG, SB, SNF, AVSH, and SJD conceived and performed the experiments. ROP, SES, SCE, KM, FG, CAL, and SJD analyzed the data. JJ, KM, EB, ASI, CMN, MB, FDP, MFO, LB, JW, LMM, FHO, JSM, AMM, ADD, GJW, and CAL contributed reagents, materials, and analysis tools. RR, RA, NKV, FLN, JV, and AML managed the project. ROP and SJD wrote the paper.

\section{Acknowledgments}

The authors are grateful for the assistance of Julie Furze, Alexandra Spencer, Joe Illingworth, Rebecca Dabbs, Bernadeta Dadonaite, Jack Quaddy, Natalie Lella, Raquel Lopez-Ramon, Megan Baker, and Charlotte Tyson (Jenner Institute, University of Oxford); Sylwester Pawluk (ITCRC); Nicola Pugh, Prudence Miyanza, Shauna Wakefield, and Filipa Martins (NIHR Wellcome Trust Clinical Research Facility, University Hospital Southampton NHS Foundation Trust); Kate Wright and Matthew Higgins (Biochemistry Department, University of Oxford); Ekta Mukhopadhyay, Richard Tarrant, and Sarah Moyle (Clinical Biomanufacturing Facility, University of Oxford); Zenon Zenonos (Wellcome Trust Sanger Institute); Amy Duckett and Carly Banner for arranging contracts (University of Oxford); the Director of KEMRI-Wellcome Trust (Kilifi, Kenya) for permission to publish the study; Sophia Hundt and Oliver Schraidt for project management support (European Vaccine Initiative); Ripley Ballou and Danielle Morelle (GSK Vaccines); Alfredo Nicosia, Stefano Colloca, Antonella Folgori, and Stefania Capone (ReiThera SRL, for- 
merly Okairos SRL); and all the study volunteers. This work is in memory of our colleague Riccardo Cortese (ReiThera SRL, formerly Okairos SRL).

This work was supported by funding from the European Union Seventh Framework Programme (FP7/20072013) under the grant agreement for MultiMalVax (number 305282). The study was also supported in part by UK NIHR infrastructure through the NIHR Oxford Biomedical Research Centre; the MAVARECA program funded by Danida (the Consultative Committee for Development Research, Denmark); and the Wellcome Trust (grant numbers $084113 / \mathrm{Z} / 07 / \mathrm{Z}$ and 206194). The GIA work was supported by the United States Agency for International Development (USAID) and the Intramural Program of the NIH, National Institute of Allergy and Infectious Diseases. DGWA holds a UK MRC iCASE PhD Studentship (MR/K017632/1); JSM is supported by a National Health and Medical Research Council (NHMRC) Practitioner Fellowship (10418020); ADD held a Wellcome Trust Training Fellowship for Clinicians in Basic Sciences (089455/2/09/z); SB, AVSH, and SJD are Jenner Investigators; and SJD is a Lister Institute Research Prize Fellow and a Wellcome Trust Senior Fellow (106917/Z/15/Z)

Address correspondence to: Ruth O. Payne or Simon J. Draper, Jenner Institute, Old Road Campus Research Building, Roosevelt Drive, Oxford, OX3 7DQ, United Kingdom. Phone: 44.1865.617624; Email: ruth.payne@ndm.ox.ac.uk (ROP), simon.draper@ndm.ox.ac.uk (SJD).

J. Vekemans present address is: Initiative for Vaccine Research, World Health Organization, Geneva, Switzerland.

1. WHO. World Malaria Report 2015. Geneva, Switzerland: World Health Organization; 2015. http://www.who.int/malaria/publications/world-malaria-report-2015/report/en/. Accessed October 10, 2017.

2. Moorthy VS, Newman RD, Okwo-Bele JM. Malaria vaccine technology roadmap. Lancet. 2013;382(9906):1700-1701.

3. Halbroth BR, Draper SJ. Recent developments in malaria vaccinology. Adv Parasitol. 2015;88:1-49.

4. RTS,S Clinical Trials Partnership. Efficacy and safety of RTS,S/AS01 malaria vaccine with or without a booster dose in infants and children in Africa: final results of a phase 3, individually randomised, controlled trial. Lancet. 2015;386(9988):31-45.

5. Sissoko MS, et al. Safety and efficacy of PfSPZ Vaccine against Plasmodium falciparum via direct venous inoculation in healthy malaria-exposed adults in Mali: a randomised, double-blind phase 1 trial. Lancet Infect Dis. 2017;17(5):498-509.

6. Langhorne J, Ndungu FM, Sponaas AM, Marsh K. Immunity to malaria: more questions than answers. Nat Immunol. 2008;9(7):725-732.

7. Takala SL, et al. Extreme polymorphism in a vaccine antigen and risk of clinical malaria: implications for vaccine development. Sci Transl Med. 2009;1(2):2ra5.

8. Wright GJ, Rayner JC. Plasmodium falciparum erythrocyte invasion: combining function with immune evasion. PLoS Pathog. 2014;10(3):e1003943.

9. Miura K, et al. Anti-apical-membrane-antigen-1 antibody is more effective than anti-42-kilodalton-merozoite-surface-protein-1 antibody in inhibiting plasmodium falciparum growth, as determined by the in vitro growth inhibition assay. Clin Vaccine Immunol. 2009;16(7):963-968.

10. Saul A. Kinetic constraints on the development of a malaria vaccine. Parasite Immunol. 1987;9(1):1-9.

11. Draper SJ, et al. Recent advances in recombinant protein-based malaria vaccines. Vaccine. 2015;33(52):7433-7443.

12. Douglas AD, et al. The blood-stage malaria antigen PfRH5 is susceptible to vaccine-inducible cross-strain neutralizing antibody. Nat Commun. 2011;2:601.

13. Reddy KS, Amlabu E, Pandey AK, Mitra P, Chauhan VS, Gaur D. Multiprotein complex between the GPI-anchored CyRPA with PfRH5 and PfRipr is crucial for Plasmodium falciparum erythrocyte invasion. Proc Natl Acad Sci U S A. 2015;112(4):1179-1184.

14. Chen L, et al. An EGF-like protein forms a complex with PfRh5 and is required for invasion of human erythrocytes by Plasmodium falciparum. PLoS Pathog. 2011;7(9):e1002199.

15. Drew DR, Beeson JG. PfRH5 as a candidate vaccine for Plasmodium falciparum malaria. Trends Parasitol. 2015;31(3):87-88.

16. Baum J, et al. Reticulocyte-binding protein homologue 5 - an essential adhesin involved in invasion of human erythrocytes by Plasmodium falciparum. Int J Parasitol. 2009;39(3):371-380.

17. Rodriguez M, Lustigman S, Montero E, Oksov Y, Lobo CA. PfRH5: a novel reticulocyte-binding family homolog of plasmodium falciparum that binds to the erythrocyte, and an investigation of its receptor. PLoS One. 2008;3(10):e3300.

18. Williams AR, et al. Enhancing blockade of Plasmodium falciparum erythrocyte invasion: assessing combinations of antibodies against PfRH5 and other merozoite antigens. PLoS Pathog. 2012;8(11):e1002991.

19. Bustamante LY, et al. A full-length recombinant Plasmodium falciparum PfRH5 protein induces inhibitory antibodies that are effective across common PfRH5 genetic variants. Vaccine. 2013;31(2):373-379.

20. Reddy KS, et al. Bacterially expressed full-length recombinant Plasmodium falciparum RH5 protein binds erythrocytes and elicits potent strain-transcending parasite-neutralizing antibodies. Infect Immun. 2014;82(1):152-164.

21. Hayton K, et al. Erythrocyte binding protein PfRH5 polymorphisms determine species-specific pathways of Plasmodium falciparum invasion. Cell Host Microbe. 2008;4(1):40-51.

22. Crosnier C, et al. Basigin is a receptor essential for erythrocyte invasion by Plasmodium falciparum. Nature. 
2011;480(7378):534-537.

23. Tran TM, et al. Naturally acquired antibodies specific for Plasmodium falciparum reticulocyte-binding protein homologue 5 inhibit parasite growth and predict protection from malaria. J Infect Dis. 2014;209(5):789-798.

24. Villasis E, et al. Anti-Plasmodium falciparum invasion ligand antibodies in a low malaria transmission region, Loreto, Peru. Malar J. 2012;11:361.

25. Richards JS, et al. Identification and prioritization of merozoite antigens as targets of protective human immunity to Plasmodium falciparum malaria for vaccine and biomarker development. J Immunol. 2013;191(2):795-809.

26. Osier FH, et al. New antigens for a multicomponent blood-stage malaria vaccine. Sci Transl Med. 2014;6(247):247ra102.

27. Hayton K, Dumoulin P, Henschen B, Liu A, Papakrivos J, Wellems TE. Various PfRH5 polymorphisms can support Plasmodium falciparum invasion into the erythrocytes of owl monkeys and rats. Mol Biochem Parasitol. 2013;187(2):103-110.

28. Wanaguru M, Liu W, Hahn BH, Rayner JC, Wright GJ. RH5-Basigin interaction plays a major role in the host tropism of Plasmodium falciparum. Proc Natl Acad Sci U S A. 2013;110(51):20735-20740.

29. Galaway F, et al. P113 is a merozoite surface protein that binds the $\mathrm{N}$ terminus of Plasmodium falciparum RH5. Nat Commun $2017 ; 8: 14333$

30. Volz JC, et al. Essential Role of the PfRh5/PfRipr/CyRPA complex during Plasmodium falciparum invasion of erythrocytes. Cell Host Microbe. 2016;20(1):60-71.

31. Aniweh Y, et al. P. falciparum RH5-Basigin interaction induces changes in the cytoskeleton of the host RBC. Cell Microbiol. 2017;19(9):e12747.

32. Draper SJ, et al. Effective induction of high-titer antibodies by viral vector vaccines. Nat Med. 2008;14(8):819-821.

33. de Cassan SC, Draper SJ. Recent advances in antibody-inducing poxviral and adenoviral vectored vaccine delivery platforms for difficult disease targets. Expert Rev Vaccines. 2013;12(4):365-378.

34. Douglas AD, et al. A PfRH5-based vaccine is efficacious against heterologous strain blood-stage Plasmodium falciparum infection in aotus monkeys. Cell Host Microbe. 2015;17(1):130-139.

35. Draper SJ, et al. Enhancing blood-stage malaria subunit vaccine immunogenicity in rhesus macaques by combining adenovirus, poxvirus, and protein-in-adjuvant vaccines. J Immunol. 2010;185(12):7583-7595.

36. O'Hara GA, et al. Clinical assessment of a recombinant simian adenovirus ChAd63: a potent new vaccine vector. J Infect Dis. 2012;205(5):772-781.

37. de Barra E, et al. A phase Ia study to assess the safety and immunogenicity of new malaria vaccine candidates ChAd63 CS administered alone and with MVA CS. PLoS One. 2014;9(12):e115161.

38. Sheehy SH, et al. Phase Ia clinical evaluation of the Plasmodium falciparum blood-stage antigen MSP1 in ChAd63 and MVA vaccine vectors. Mol Ther. 2011;19(12):2269-2276.

39. Sheehy SH, et al. Phase Ia clinical evaluation of the safety and immunogenicity of the Plasmodium falciparum blood-stage antigen AMA1 in ChAd63 and MVA vaccine vectors. PLoS One. 2012;7(2):e31208.

40. Sedegah M, et al. Adenovirus 5-vectored P. falciparum vaccine expressing CSP and AMA1. Part A: safety and immunogenicity in seronegative adults. PLoS One. 2011;6(10):e24586.

41. Afolabi MO, et al. Safety and immunogenicity of ChAd63 and MVA ME-TRAP in West African children and infants. Mol Ther. 2016;24(8):1470-1477.

42. Kimani D, et al. Translating the immunogenicity of prime-boost immunization with ChAd63 and MVA ME-TRAP from malaria naive to malaria-endemic populations. Mol Ther. 2014;22(11):1992-2003.

43. Payne RO, et al. Human vaccination against Plasmodium vivax Duffy-binding protein induces strain-transcending antibodies. JCI Insight. 2017;2(12):93683.

44. Ewer K, et al. A Monovalent Chimpanzee Adenovirus Ebola Vaccine Boosted with MVA. N Engl J Med. 2016;374(17):1635-1646.

45. Barnes E, et al. Novel adenovirus-based vaccines induce broad and sustained T cell responses to HCV in man. Sci Transl Med. 2012;4(115):115ra1.

46. Green CA, et al. Chimpanzee adenovirus- and MVA-vectored respiratory syncytial virus vaccine is safe and immunogenic in adults. Sci Transl Med. 2015;7(300):300ra126.

47. Borthwick N, et al. Vaccine-elicited human T cells recognizing conserved protein regions inhibit HIV-1. Mol Ther. 2014;22(2):464-475.

48. Sheehy SH, et al. Phase Ia clinical evaluation of the Plasmodium falciparum blood-stage antigen MSP1 in ChAd63 and MVA vaccine vectors. Mol Ther. 2011;19(12):2269-2276.

49. Wright KE, et al. Structure of malaria invasion protein RH5 with erythrocyte basigin and blocking antibodies. Nature. 2014;515(7527):427-430.

50. Chen L, et al. Crystal structure of PfRh5, an essential $P$. falciparum ligand for invasion of human erythrocytes. Elife. 2014;3:e04187.

51. Elias SC, et al. Analysis of human B-cell responses following ChAd63-MVA MSP1 and AMA1 immunization and controlled malaria infection. Immunology. 2014;141(4):628-644.

52. Hodgson $\mathrm{SH}$, et al. Combining viral vectored and protein-in-adjuvant vaccines against the blood-stage malaria antigen AMA1: report on a phase 1a clinical trial. Mol Ther. 2014;22(12):2142-2154.

53. Payne RO, et al. Demonstration of the blood-stage Plasmodium falciparum controlled human malaria infection model to assess efficacy of the P. falciparum apical membrane antigen 1 vaccine, FMP2.1/AS01. J Infect Dis. 2016;213(11):1743-1751.

54. Sheehy SH, et al. ChAd63-MVA-vectored blood-stage malaria vaccines targeting MSP1 and AMA1: assessment of efficacy against mosquito bite challenge in humans. Mol Ther. 2012;20(12):2355-2368.

55. Stanisic DI, et al. Infectivity of Plasmodium falciparum in malaria-naive individuals is related to knob expression and cytoadherence of the parasite. Infect Immun. 2016;84(9):2689-2696.

56. Douglas AD, et al. Neutralization of Plasmodium falciparum merozoites by antibodies against PfRH5. J Immunol. 2014;192(1):245-258.

57. Gilbert SC. Clinical development of modified vaccinia virus Ankara vaccines. Vaccine. 2013;31(39):4241-4246.

58. Swadling L, et al. A human vaccine strategy based on chimpanzee adenoviral and MVA vectors that primes, boosts, and sus- 
tains functional HCV-specific T cell memory. Sci Transl Med. 2014;6(261):261ra153.

59. Bijker EM, et al. Protection against malaria after immunization by chloroquine prophylaxis and sporozoites is mediated by preerythrocytic immunity. Proc Natl Acad Sci U S A. 2013;110(19):7862-7867.

60. Draper SJ, et al. Recombinant viral vaccines expressing merozoite surface protein-1 induce antibody- and T cell-mediated multistage protection against malaria. Cell Host Microbe. 2009;5(1):95-105.

61. Vargas-Inchaustegui DA, et al. Vaccine induction of lymph node-resident simian immunodeficiency virus Env-specific T follicular helper cells in rhesus macaques. J Immunol. 2016;196(4):1700-1710.

62. Wang C, et al. Germinal center B cell and T follicular helper cell responses to viral vector and protein-in-adjuvant vaccines. $J$ Immunol. 2016;197(4):1242-1251.

63. Biswas S, et al. Assessment of humoral immune responses to blood-stage malaria antigens following ChAd63-MVA immunization, controlled human malaria infection and natural exposure. PLoS One. 2014;9(9):e107903.

64. Weaver R, et al. The association between naturally acquired IgG subclass specific antibodies to the PfRH5 invasion complex and protection from Plasmodium falciparum malaria. Sci Rep. 2016;6:33094.

65. Boyle MJ, et al. Human antibodies fix complement to inhibit Plasmodium falciparum invasion of erythrocytes and are associated with protection against malaria. Immunity. 2015;42(3):580-590.

66. Manske M, et al. Analysis of Plasmodium falciparum diversity in natural infections by deep sequencing. Nature. 2012;487(7407):375-379.

67. Ord RL, et al. A malaria vaccine candidate based on an epitope of the Plasmodium falciparum RH5 protein. Malar J. 2014;13:326.

68. Chen L, et al. Structural basis for inhibition of erythrocyte invasion by antibodies to Plasmodium falciparum protein CyRPA. Elife. 2017;6:e21347.

69. Hjerrild KA, et al. Production of full-length soluble Plasmodium falciparum RH5 protein vaccine using a Drosophila melanogaster Schneider 2 stable cell line system. Sci Rep. 2016;6:30357.

70. Malkin EM, et al. Phase 1 clinical trial of apical membrane antigen 1: an asexual blood-stage vaccine for Plasmodium falciparum malaria. Infect Immun. 2005;73(6):3677-3685.

71. Bushell KM, Söllner C, Schuster-Boeckler B, Bateman A, Wright GJ. Large-scale screening for novel low-affinity extracellular protein interactions. Genome Res. 2008;18(4):622-630 